\title{
Distributions on Minkowski Space and Their Connection with Analytic Representations of the Conformal Group
}

\author{
W. RÜHL \\ Universität Trier-Kaiserslautern, Fachbereich Physik, Kaiserslautern
}

Received March 16, 1972

\begin{abstract}
Unitary analytic representations of the conformal group are realized on Hilbert spaces of holomorphic or antiholomorphic functions over a tube domain in complex Minkowski space. The distributional boundary values of these functions are tempered distributions on real Minkowski space. The representations are characterized by an integral scale dimension label $n$ and two spin labels $j_{1}$ and $j_{2}$. The connection between the dimension $n$ and the degree of singularity of the tempered distribution is investigated. We propose an application to inclusive reactions of elementary particles.
\end{abstract}

\section{Introduction and Summary}

We study the connection of unitary analytic representations of the conformal group $S U(2,2)$ with distributions on Minkowski space. The unitary representations we have in mind are realized on Hilbert spaces of functions that are holomorphic (or antiholomorphic) over the tube domain in Minkowski space. This is a manifold of vectors in complex Minkowski space whose real part is arbitrary and whose imaginary part lies in the forward light cone. Each representation of this series is characterized by a "scale dimension" $n$ that is an integer, and two spin labels $j_{1}$ and $j_{2}$. We are mainly concerned with the case $j_{1}=j_{2}=0$. The general case is algebraically more complicated, though in principle our approach applies to arbitrary spins.

Any vector of such Hilbert space possesses a tempered distribution as distributional boundary value on Minkowski space. In turn, any tempered distribution that can be regarded as a Fourier transform of a tempered distribution with support in the forward light cone, is the distributional boundary value of a holomorphic function which belongs to a Hilbert space carrying one of the representations of the series considered. Our aim is to characterize the scale dimension $n$ of the representation by the degree of the singularities of the tempered distribution. 
We define first the unitary representations of $S U(2,2)$ on a compact realization of the tube domain. This allows us to use a polynomial basis in the Hilbert spaces, and with its help to construct the Bergman kernel explicitly. The Bergman kernel majorizes the polynomial increase of any holomorphic function of a given Hilbert space at the boundary of the domain. Thus it determines an upper bound for the degree of the singularity which the distributional boundary value may assume. In turn we define generalized Fourier series on the Shilov boundary of the compact domain. We show how these can be extended into the interior of the domain. The scale dimension $n$ is connected with the polynomial order of increase of the coefficients of the Fourier series. Another technique for extending distributions from the Shilov boundary into the interiour makes use of the Szegö kernel. It enables us to derive another (less restrictive) estimate of the scale dimension.

The compact realization is mapped onto the noncompact tube domain in complex Minkowski space by a matrix transformation of Cayley type. All technical devices like the invariant scalar product, the Bergman and the Szegö kernels are carried over. The new Szegö kernel is essentially the Fourier transformed characteristic function of the forward light cone. Since we have no natural Fourier expansion in the noncompact realization, we use the Szegö kernel to estimate the scale dimension of the holomorphic extensions.

Finally we sketch some ideas of how this formalism could be applied to physics. An application to inclusive reactions of elementary particles seems to us most interesting. Any a priori (say from field theory) or phenomenological information on the scale dimension $n$ can be used to deduce the convergence of an integral over structure functions that is identical with the conformally invariant scalar product.

\section{The Conformal Group}

\subsection{The Definition of the Conformal Group}

We define the conformal group $S U(2,2)$ as follows. We consider complex $4 \times 4$ matrices

$$
M=\left(\begin{array}{ll}
A & B \\
C & D
\end{array}\right)
$$

where $A, B, C, D$ are $2 \times 2$ submatrices. The matrix $M$ is assumed to satisfy the constraint

with

$$
M^{+} H=H M^{-1}
$$

$$
H=\left(\begin{array}{rr}
-E & 0 \\
0 & E
\end{array}\right)
$$


and the $2 \times 2$ unit matrix $E$. The constraint (1.2) is equivalent with the set of three relations

$$
\begin{aligned}
& A^{+} A-C^{+} C=E \\
& D^{+} D-B^{+} B=E \\
& A^{+} B-C^{+} D=0 .
\end{aligned}
$$

The group resulting from the constraint (1.2) is denoted $U(2,2)$. If the further constraint det $M=1$ is fulfilled, we obtain the group $S U(2,2)$.

An immediate consequence of (1.4) is that $A$ and $D$ possess inverses. By some elementary algebra one can show that (1.4) is equivalent with the set of constraints.

$$
\begin{aligned}
& A A^{+}-B B^{+}=E \\
& D D^{+}-C C^{+}=E \\
& A C^{+}-B D^{+}=0 .
\end{aligned}
$$

\subsection{The Maximal Compact Subgroup and Its Coset Space}

The maximal compact subgroup of $S U(2,2)$ consists of the matrices

$$
\left(\begin{array}{ll}
K_{1} & 0 \\
0 & K_{2}
\end{array}\right), \quad K_{1,2} \in U(2)
$$

with the constraint

$$
\operatorname{det}\left(K_{1} K_{2}\right)=1 .
$$

It has the direct product structure $U(2) \otimes S U(2)$. Each matrix $M$ of $S U(2,2)$ can be uniquely decomposed in the fashion

$$
\left(\begin{array}{ll}
A & B \\
C & D
\end{array}\right)=\left(\begin{array}{ll}
N_{1}, & Z N_{2} \\
Z^{+} N_{1}, & N_{2}
\end{array}\right)\left(\begin{array}{ll}
K_{1} & 0 \\
0 & K_{2}
\end{array}\right)
$$

where both matrices on the right-hand-side are in $S U(2,2)$. We want $N_{1}$ and $N_{2}$ to be positive definite, hermitean matrices. $Z$ is an arbitrary complex $2 \times 2$ matrix.

We consider (1.8) as an ansatz. Then

$$
\begin{array}{ll}
A=N_{1} K_{1}, & B=Z N_{2} K_{2}, \\
C=Z^{+} N_{1} K_{1}, & D=N_{2} K_{2} .
\end{array}
$$

Since the inverses of $A$ and $D$ exist, we have necessarily

$$
\begin{aligned}
Z & =B D^{-1} \\
Z^{+} & =C A^{-1} .
\end{aligned}
$$


The compatibility of these two definitions of $Z$ is equivalent with the last constraint (1.4). With $Z$ defined by (1.10), $N_{1}$ and $N_{2}$ follow from (1.9)

$$
\begin{gathered}
N_{1}=\left(A A^{+}\right)^{\frac{1}{2}} \\
N_{2}=\left(D D^{+}\right)^{\frac{1}{2}} .
\end{gathered}
$$

As required they are positive definite and hermitean.

We still have to satisfy the other two constraints (1.4). First we have from (1.11) and (1.9) that

$$
K_{1}=N_{1}^{-1} A, \quad K_{2}=N_{2}^{-1} D
$$

are unitary. This is the polar decomposition. Next it follows from (1.9) and (1.4)

$$
\begin{aligned}
& N_{1}^{2}-N_{1} Z Z^{+} N_{1}=E \\
& N_{2}^{2}-N_{2} Z^{+} Z N_{2}=E .
\end{aligned}
$$

These equations for $N_{1.2}$ can be solved to give

$$
\begin{aligned}
& N_{1}^{-2}=E-Z Z^{+} \\
& N_{2}^{-2}=E-Z^{+} Z .
\end{aligned}
$$

It follows that $Z$ satisfies the conditions

$$
\begin{aligned}
& E-Z Z^{+}>0 \\
& E-Z^{+} Z>0
\end{aligned}
$$

that are in fact equivalent.

We have finally to show that the constraint (1.7) is satisfied. For this purpose we decompose $Z$ as

$$
Z=u_{1}\left(\begin{array}{ll}
\lambda_{1} & 0 \\
0 & \lambda_{2}
\end{array}\right) u_{2}
$$

where $u_{1,2} \in S U(2)$ and $\lambda_{1,2}$ are complex numbers. This decomposition allows us to write $N_{1}$ and $N_{2}$ as

$$
N_{1}=u_{1}\left(\begin{array}{ll}
\mu_{1} & 0 \\
0 & \mu_{2}
\end{array}\right) u_{1}^{-1}, \quad N_{2}=u_{2}^{-1}\left(\begin{array}{cc}
\mu_{1} & 0 \\
0 & \mu_{2}
\end{array}\right) u_{2}, \quad \mu_{1,2}=\left(1-\left|\lambda_{1,2}\right|^{2}\right)^{-\frac{1}{2}}
$$

so that

$$
\left(\begin{array}{ll}
N_{1}, & Z N_{2} \\
Z^{+} N_{1}, & N_{2}
\end{array}\right)=\left(\begin{array}{ll}
u_{1} & 0 \\
0 & u_{2}^{-1}
\end{array}\right)\left(\begin{array}{llll}
\mu_{1} & 0 & \lambda_{1} \mu_{1} & 0 \\
0 & \mu_{2} & 0 & \lambda_{2} \mu_{2} \\
\lambda_{1} \mu_{1} & 0 & \mu_{1} & 0 \\
0 & \bar{\lambda}_{2} \mu_{2} & 0 & \mu_{2}
\end{array}\right)\left(\begin{array}{ll}
u_{1}^{-1} & 0 \\
0 & u_{2}
\end{array}\right) .
$$


The determinant of this matrix is easily computed

$$
\operatorname{det}\left(\begin{array}{ll}
N_{1}, & Z N_{2} \\
Z^{+} N_{1}, & N_{2}
\end{array}\right)=\mu_{1} \mu_{2} \mu_{1}\left(1-\left|\lambda_{1}\right|^{2}\right) \mu_{2}\left(1-\left|\lambda_{2}\right|^{2}\right)=1
$$

Therefore the constraint (1.7) is fulfilled.

It results that the cosets of the maximal compact subgroup $U(2) \times S U(2)$ can be mapped one-to-one on the domain of complex $2 \times 2$ matrices $Z$ that satisfy

$$
E-Z^{+} Z>0
$$

We call this domain the compact realization of the coset space and denote it by $\mathbb{D}$.

\subsection{Left Translations on the Coset Space and Unitary Representations}

In this section we denote the inverse $M^{-1}$ of any $M \in S U(2,2)$ by

$$
M^{-1}=\left(\begin{array}{ll}
A & B \\
C & D
\end{array}\right)
$$

Then the left translation by $M$ maps the coset space onto itself and the explicit form of this mapping can be computed from

$$
\left(\begin{array}{ll}
A & B \\
C & D
\end{array}\right)\left(\begin{array}{ll}
N_{1}, & Z N_{2} \\
Z^{+} N_{1}, & N_{2}
\end{array}\right)=\left(\begin{array}{ll}
N_{1}^{\prime}, & Z^{\prime} N_{2}^{\prime} \\
Z^{\prime+} N_{1}^{\prime}, & N_{2}^{\prime}
\end{array}\right)\left(\begin{array}{ll}
K_{1}^{\prime} & 0 \\
0 & K_{2}^{\prime}
\end{array}\right) .
$$

It results

$$
Z^{\prime}=(A Z+B)(C Z+D)^{-1} .
$$

The arguments of the preceding section guarantee that the inverse of $C Z+D$ exists over $\mathbb{D}$.

The Lebesgue measure on $\mathbb{D}$ is defined by

$$
Z=\left(\begin{array}{ll}
z_{11}, & z_{12} \\
z_{21}, & z_{22}
\end{array}\right), \quad|d Z|=\prod_{i, j=1.2} d \operatorname{Re} z_{i j} d \operatorname{Im} z_{i j} .
$$

For arbitrary $n=4,5,6, \ldots$ we define the Hilbert space $\mathscr{L}_{n}^{2}(\mathbb{D})$ by

$$
\mathscr{L}_{n}^{2}(\mathbb{D})=\left\{f(Z) \mid f(Z) \text { measurable on } \mathbb{D} \text { and }\|f\|_{n}<\infty\right\}
$$

where the norm (and a corresponding scalar product) is defined by

$$
\|f\|_{n}^{2}=c \int_{\mathbb{D}}|f(Z)|^{2}\left[\operatorname{det}\left(E-Z^{+} Z\right)\right]^{n-4}|d Z| .
$$

The normalization constant $c$ is positive and is later fixed in such a fashion that the norm of $f(Z)=1$ is one. 
In the space $\mathscr{L}_{n}^{2}(\mathbb{D})$ we define the unitary representation $T$ by

$$
T_{M} f(Z)=[\operatorname{det}(C Z+D)]^{-n} f\left(Z^{\prime}\right)
$$

where $Z^{\prime}$ is as in (1.23) and $M$ as in (1.21). The unitarity of $T_{M}$ and the operator relation

$$
T_{M_{1}} T_{M_{2}}=T_{M_{1} M_{2}}
$$

have yet to be proved.

In fact, from (1.4) and (1.23) we obtain

and

$$
E-Z^{+} Z^{\prime}=(C Z+D)^{-1+}\left(E-Z^{+} Z\right)(C Z+D)^{-1}
$$

$$
|d Z|=\left|d Z^{\prime}\right||\operatorname{det}(C Z+D)|^{8} .
$$

This gives immediately the isometry relation

$$
\left\|T_{M} f\right\|_{n}^{2}=\|f\|_{n}^{2}
$$

that together with (1.28) for $M_{2}=M_{1}^{-1}$ implies the unitarity of $T_{M}$. In order to prove (1.28) we put

and get

$$
\tilde{f}=T_{M_{2}} f, \quad Z_{1}=\left(A_{1} Z+B_{1}\right)\left(C_{1} Z+D_{1}\right)^{-1}
$$

$$
\begin{aligned}
T_{M_{1}} & \left(T_{M_{2}} f\right)(Z)=T_{M_{1}} \tilde{f}(Z) \\
\quad & =\left[\operatorname{det}\left(C_{1} Z+D_{1}\right)\right]^{-n} \tilde{f}\left(Z_{1}\right) \\
& =\left[\operatorname{det}\left(C_{1} Z+D_{1}\right) \operatorname{det}\left(C_{2} Z_{1}+D_{2}\right)\right]^{-n} f\left(\left(A_{2} Z_{1}+B_{2}\right)\left(C_{2} Z_{1}+D_{2}\right)^{-1}\right) .
\end{aligned}
$$

Now with

$$
\left(M_{1} M_{2}\right)^{-1}=M_{3}^{-1}=\left(\begin{array}{ll}
A_{2} & B_{2} \\
C_{2} & D_{2}
\end{array}\right)\left(\begin{array}{ll}
A_{1} & B_{1} \\
C_{1} & D_{1}
\end{array}\right)=\left(\begin{array}{ll}
A_{3} & B_{3} \\
C_{3} & D_{3}
\end{array}\right)
$$

we obtain by elementary algebra

and

$$
\left(C_{2} Z_{1}+D_{2}\right)\left(C_{1} Z+D_{1}\right)=\left(C_{3} Z+D_{3}\right)
$$

$$
\left(A_{2} Z_{1}+B_{2}\right)\left(C_{2} Z_{1}+D_{2}\right)^{-1}=\left(A_{3} Z+B_{3}\right)\left(C_{3} Z+D_{3}\right)^{-1}
$$

so that (1.28) follows.

The space $\mathscr{L}_{n}^{2}(\mathbb{D})$ possesses two invariant subspaces: The space $\mathscr{H}_{n}(\mathbb{D})$ of holomorphic functions in $\mathbb{D}$ and the space $\mathscr{H}_{n}^{*}(\mathbb{D})$ of antiholomorphic functions in $\mathbb{D}$. The restriction of $T$ to these subspaces gives irreducible unitary representations of $S U(2,2)$. In Graev's classification $[1,2]$ these representations belong to the series $d_{0}$. A general member of this series is characterized by two additional spin labels $j_{1}$ and $j_{2}$ (see Section 3.1). The representations just constructed and mainly 
studied in this work have $j_{1}=j_{2}=0$. A generalization of our arguments to all spins is straightforward in principle but in practice beset with computational complications.

\subsection{An Orthonormal Basis}

The set of all polynomials in the matrix elements of $Z$ forms a complete system of functions in $\mathscr{H}_{n}(\mathbb{D})$. By a convenient choice of these functions and the Schmidt orthogonalization procedure one can construct an orthonormal basis in $\mathscr{H}_{n}(\mathbb{D})$. We shall construct such a basis now. The corresponding complex conjugate functions form a basis in $\mathscr{H}_{n}^{*}(\mathrm{D})$.

We introduce first a set of convenient parameters in $\mathbb{D}$. The decomposition (1.16) of $Z$ can obviously be done such that $u_{1}$ and $u_{2}$ assume the form

$$
\begin{aligned}
& u_{1}=e^{i \varphi_{1} \sigma_{3}} e^{l \vartheta_{1} \sigma_{2}} \\
& u_{2}=e^{i \vartheta_{2} \sigma_{2}} e^{i \varphi_{2} \sigma_{3}}
\end{aligned}
$$

with Pauli matrices $\sigma_{2}, \sigma_{3}$. They define cosets in $S U(2)$. On these cosets we introduce the normalized measures

$$
\begin{aligned}
d^{\prime} \mu\left(u_{1}\right) & =\frac{1}{2 \pi} d \varphi_{1} d t_{1} \\
d^{\prime} \mu\left(u_{2}\right) & =\frac{1}{2 \pi} d \varphi_{2} d t_{2} \\
0 \leqq \varphi_{1,2} \leqq 2 \pi, \quad t_{1,2} & =\cos ^{2} \vartheta_{1,2}, \quad 0 \leqq t_{1,2} \leqq 1 .
\end{aligned}
$$

We denote the Lebesgue measure in the complex plane by

$$
|d \lambda|=d \operatorname{Re} \lambda d \operatorname{Im} \lambda .
$$

With the parameters appearing in the decomposition (1.16) we have

$$
|d Z|=J d^{\prime} \mu\left(u_{1}\right) d^{\prime} \mu\left(u_{2}\right)\left|d \lambda_{1}\right|\left|d \lambda_{2}\right|
$$

After some algebra we find

$$
J=\frac{1}{2} \pi^{2}\left(\left|\lambda_{1}\right|^{2}-\left|\lambda_{2}\right|^{2}\right)^{2} .
$$

As a comfortable check of the normalization of the functional determinant (1.41) one can compute the integral

$$
V_{8}=\int \theta\left(1-\operatorname{Tr}\left(Z^{+} Z\right)\right)|d Z|=\frac{\pi^{4}}{24}
$$

where $V_{n}$ denotes the volume of the unit sphere in $n$-dimensional real Euclidean space. With the parameters (1.16) and polar coordinates 
for $\lambda_{1}$ and $\lambda_{2}$ the integral (1.42) reduces to a two-dimensional elementary integral.

We introduce the set of polynomials

$$
\begin{gathered}
\Delta_{q_{1} q_{2}}^{j m}(Z)=\left(N^{j m}\right)^{-1}(\operatorname{det} Z)^{m} D_{q_{1} q_{2}}^{j}(Z) \\
m=0,1,2, \ldots, \quad-j \leqq q_{1}, q_{2} \leqq+j, \quad 2 j=0,1,2, \ldots
\end{gathered}
$$

where $N^{j m}$ is a normalization factor that renders the norm of these functions equal to one. The polynomials $D_{q_{1} q_{2}}^{j}$ are known from the theory of the representations of $S U(2)$ and are defined by [3]

$$
\begin{aligned}
D_{q_{1} q_{2}}^{j}(Z)= & {\left[\frac{\left(j+q_{1}\right) !\left(j-q_{1}\right) !}{\left(j+q_{2}\right) !\left(j-q_{2}\right) !}\right]^{\frac{1}{2}} \sum_{S}\left(\begin{array}{c}
j+q_{2} \\
S
\end{array}\right)\left(\begin{array}{l}
j-q_{2} \\
S-q_{1}-q_{2}
\end{array}\right) } \\
& \cdot Z_{11}^{S} Z_{12}^{j+q_{1}-S} Z_{21}^{j+q_{2}-S} Z_{22}^{S-q_{1}-q_{2}} .
\end{aligned}
$$

The polynomials (1.43) are homogeneous of degree

$$
N=2 j+2 m
$$

in the elements of $Z$. For fixed $N$ there are

$$
S_{N}=\frac{1}{6}(N+1)(N+2)(N+3)
$$

of such polynomials (1.43). It is easy to see that these polynomials are orthogonal and consequently linearly independent. On the other hand there exist just $S_{N}$ linearly independent polynomials of the type

$$
z_{11}^{n_{11}} z_{12}^{n_{12}} z_{21}^{n_{21}} z_{2}^{n_{22}}
$$

of fixed degree $N=\sum n_{i j}$ of homogeneity. Hence the polynomials (1.43) form an orthogonal basis in $\mathscr{H}_{n}(\mathbb{D})$.

Computation of the norm $N^{j m}$ yields

$$
\left(N^{j m}\right)^{2}=c \pi^{4} \frac{(n-3) !(n-4) ! m !(m+2 j+1) !}{(2 j+1)(m+n-2) !(m+2 j+n-1) !} .
$$

In order to have $N^{00}=1$ (see the remark after (1.26)) we set

$$
c=\pi^{-4}(n-1)(n-2)^{2}(n-3) \text {. }
$$

With this normalization $N^{j m}$ and $\Delta_{q_{1} q_{2}}^{j m}(Z)$ are defined for all $n \geqq 2$. We use this fact to extend our definition of the spaces $\mathscr{H}_{n}(\mathbb{D})$ and $\mathscr{H}_{n}^{*}(\mathbb{D})$ to include the numbers $n=2$ and $n=3$. We may first introduce them formally as spaces of $l^{2}$-summable sequences $\left\{a_{q_{1} q_{2}}^{j m}\right\}$. Then we define for any finite sum

$$
\left\|\sum_{j m q_{1} q_{2}} a_{q_{1} q_{2}}^{j m} \Delta_{q_{1} q_{2}}^{j m}(Z)\right\|_{n}^{2}=\sum_{j m q_{1} q_{2}}\left|a_{q_{1} q_{2}}^{j m}\right|^{2} .
$$


By Schwarz's inequality we have

$$
\left|\sum_{j m q_{1} q_{2}} a_{q_{1} q_{2}}^{j m} \Delta_{q_{1} q_{2}}^{j m}(Z)\right|^{2} \leqq\left(\sum_{j m q_{1} q_{2}}\left|a_{q_{1} q_{2}}^{j m}\right|^{2}\right)\left(\sum_{j m q_{1} q_{2}}\left|\Delta_{q_{1} q_{2}}^{j m}(Z)\right|^{2}\right) .
$$

In the subsequent section we shall see that the right most sum in (1.51) converges uniformly on any compact subset of $\mathbb{D}$. Therefore the elements of $\mathscr{H}_{n}(\mathbb{D})$ that were introduced as $l^{2}$-summable sequences of the coefficients $a_{q_{1} q_{2}}^{j m}$ correspond to absolutely and uniformly convergent series and hence to holomorphic functions in $\mathbb{D}$. Finally we extend the definition (1.50) to the whole Hilbert space.

\subsection{The Bergman Kernel Function}

In the Hilbert spaces $\mathscr{H}_{n}(\mathbb{D})$ and $\mathscr{H}_{n}^{*}(\mathbb{D})$ the unit operator can be represented as an integral operator whose kernel is called the Bergman kernel [4]. If $\left(f_{1}, f_{2}\right)_{n}$ denotes the scalar product

$$
\left(f_{1}, f_{2}\right)_{n}=c \int_{\mathbb{I}} \overline{f_{1}(Z)} f_{2}(Z)\left[\operatorname{det}\left(E-Z^{+} Z\right)\right]^{n-4}|d Z|
$$

and

$$
K^{B}\left(Z_{1}, Z_{2}\right)=K_{Z_{1}}^{B}\left(Z_{2}\right)
$$

the Bergman kernel function, then this Bergman kernel is defined such that

$$
\begin{array}{ll}
\left(\overline{K_{Z}^{B}}, f\right)_{n}=f(Z), \quad f \in \mathscr{H}_{n}(\mathbb{D}) \\
\left(K_{Z}^{B}, f\right)_{n}=f(Z), \quad f \in \mathscr{H}_{n}^{*}(\mathbb{D}) .
\end{array}
$$

By Schwarz's inequality we obtain from (1.54)

$$
\|f\|_{n}\left\|K_{Z}^{B}\right\|_{n} \geqq|f(Z)| \text {. }
$$

From the hermiticity of the kernel and (1.54) we have

$$
\left\|K_{Z}^{B}\right\|_{n}=K^{B}(Z, Z)^{\frac{1}{2}} \text {. }
$$

This can be inserted into (1.55) and gives an estimate for $f(Z)$. The normalization (1.49) of the scalar product implies

$$
K^{B}(0,0)=1 \text {. }
$$

On the space $\mathscr{L}_{n}^{2}(\mathbb{D})$ the Bergman kernel defines a projection operator onto the subspace $\mathscr{H}_{n}(\mathbb{D})$, respectively $\mathscr{H}_{n}^{*}(\mathbb{D})$, namely $f \in \mathscr{L}_{n}^{2}(\mathbb{D})$,

$$
\begin{aligned}
& f_{1}(Z)=\left(\overline{K_{Z}^{B}}, f\right)_{n} \in \mathscr{H}_{n}(\mathbb{D}) \\
& f_{2}(Z)=\left(K_{Z}^{B}, f\right)_{n} \in \mathscr{H}_{n}^{*}(\mathbb{D}) .
\end{aligned}
$$


We compute the Bergman kernel for arbitrary $n \geqq 2$. With the orthonormal basis (1.43) we have

$$
\begin{aligned}
K^{B}\left(Z_{1}, Z_{2}\right) & =\sum_{j m q_{1} q_{2}} \Delta_{q_{1} q_{2}}^{j m}\left(Z_{1}\right) \overline{\Delta_{q_{1} q_{2}}^{j m}\left(Z_{2}\right)} \\
& =\sum_{j m}\left(N^{j m}\right)^{-2}\left[\operatorname{det}\left(Z_{1} Z_{2}^{+}\right)\right]^{m} \operatorname{Tr}\left(D^{j}\left(Z_{1} Z_{2}^{+}\right)\right) .
\end{aligned}
$$

For almost all $Z_{1} Z_{2}^{+}$we can perform the decomposition

$$
Z_{1} Z_{2}^{+}=S\left(\begin{array}{cc}
\lambda_{1} & 0 \\
0 & \lambda_{2}
\end{array}\right) S^{-1}, \quad S \in S L(2, C)
$$

such that both eigenvalues are in the open unit circle. In fact, if we had

$$
Z_{1} Z_{2}^{+} x=\lambda x, \quad|\lambda| \geqq 1
$$

then with $Z_{1,2} \in \mathbb{D}$ and the shorthand $y=\lambda^{-1} Z_{2}^{+} x$ it would follow

$$
0<\left(x,\left(E-Z_{2} Z_{2}^{+}\right) x\right)=\left(y,\left(Z_{1}^{+} Z_{1}-|\lambda|^{2} E\right) y\right)<0 .
$$

Inserting (1.60) into (1.59) and using (1.43), (1.44), (1.48), (1.49) we have

$$
\begin{aligned}
K^{B}\left(Z_{1}, Z_{2}\right)= & \sum_{j m}(2 j+1) \frac{(m+n-2) !(m+2 j+n-1) !}{(n-1) !(n-2) ! m !(m+2 j+1) !} \\
& \cdot\left(\lambda_{1} \lambda_{2}\right)^{m} \frac{\lambda_{1}^{2 j+1}-\lambda_{2}^{2 j+1}}{\lambda_{1}-\lambda_{2}} .
\end{aligned}
$$

This series can be summed. It converges absolutely if both $\lambda_{1,2}$ stay inside the unit circle and yields

$$
\begin{aligned}
K^{B}\left(Z_{1}, Z_{2}\right) & =\left[\left(1-\lambda_{1}\right)\left(1-\lambda_{2}\right)\right]^{-n} \\
& =\left[1-\operatorname{Tr}\left(Z_{1} Z_{2}^{+}\right)+\operatorname{det}\left(Z_{1} Z_{2}^{+}\right)\right]^{-n} \\
& =\left[\operatorname{det}\left(E-Z_{1} Z_{2}^{+}\right)\right]^{-n} .
\end{aligned}
$$

For $Z_{1}$ and $Z_{2}$ both in compact subsets of $\mathbb{D}$ we find absolute and uniform convergence of the series (1.59). This proves the assertion made after (1.51).

The estimate (1.55) can now be given the explicit form

$$
|f(Z)| \leqq\|f\|_{n}\left[\operatorname{det}\left(E-Z Z^{+}\right)\right]^{-\frac{n}{2}}
$$

Consequently the elements of $\mathscr{H}_{n}(\mathbb{D})$ and $\mathscr{H}_{n}^{*}(\mathbb{D})$ increase at most polynomially if $Z$ tends to the boundary $\partial \mathbb{D}$ of $\mathbb{D}$. According to a general theory of boundary values of analytic functions the boundary values are distributions of a certain type $[5,6]$. We are mainly concerned with these boundary value distributions in the sequel. 


\subsection{The Shilov Boundary}

The boundary $\hat{\partial} \mathbb{D}$ of $\mathbb{D}$ is given by those matrices $Z$ for which in the decomposition (1.16) either $\left|\lambda_{1}\right|=1$ or $\left|\lambda_{2}\right|=1$. This is a seven dimensional manifold. In order to characterize analytic functions on $\mathbb{D}$ it suffices, however, to give their boundary values on the Shilov boundary [7]. The Shilov boundary $\mathbb{S}$ of $\mathbb{D}$ consists of all unitary matrices

$$
\mathbb{S}=\{Z \mid Z \in U(Z)\} .
$$

We denote the elements of $\$$ by $X$. Then

$$
X=e^{\frac{1}{2} \varphi} u, \quad u \in S U(2) .
$$

We introduce the normalized measure on $\mathbb{S}$

$$
\begin{gathered}
d \mu(X)=\frac{1}{2 \pi} d \varphi d \mu(u) \\
0 \leqq \varphi \leqq 2 \pi, \quad \int d \mu(u)=1
\end{gathered}
$$

where $d \mu(u)$ is the normalized Haar measure on $S U(2)$. As one possible set of parameters we may use

$$
u=\left(\begin{array}{rr}
\alpha+i \beta & \gamma+i \delta \\
-\gamma+i \delta & \alpha-i \beta
\end{array}\right)
$$

Due to the constraint

$$
\alpha^{2}+\beta^{2}+\gamma^{2}+\delta^{2}=1
$$

only three of these parameters are independent. It is easy to verify that the Haar measure on $S U(2)$ in these parameters assumes the form

$$
d \mu(u)=\frac{1}{\Omega_{4}} d \Omega_{4}
$$

where $d \Omega_{4}$ is the Lebesgue measure on the surface of the unit sphere in four dimensional Euclidean space, $\Omega_{4}$ is the total area of this surface, $\Omega_{4}=2 \pi^{2}$.

We consider measurable and square integrable functions $g(X)$ on $\mathbb{\$}$. They constitute the Hilbert space $\mathscr{L}^{2}(\mathbb{\$})$. These functions can be expanded into a generalized Fourier series

$$
g(X)=\sum_{m=-\infty}^{+\infty} \sum_{j=0}^{\infty} \sum_{q_{1} q_{2}=-j}^{+j} a_{q_{1} q_{2}}^{j m}(2 j+1)^{\frac{1}{2}} e^{i(m+j) \varphi} D_{q_{1} q_{2}}^{j}(u)
$$

where $m$ runs over all integers and $2 j$ over the nonnegative integers as usual. Half integral $m$ do not occur since the substitution

$$
u \rightarrow-u, \quad \varphi \rightarrow \varphi+2 \pi
$$


must leave both sides of (1.72) unchanged. The series (1.72) converges in the $\mathscr{L}^{2}(\mathbb{S})$ norm sense.

Restricting the summation in (1.72) to subsets we define the following new functions (the "positive, negative, and neutral parts" of $g(X)$ )

$$
\begin{aligned}
g_{+}(X) & =\sum_{j=0}^{\infty} \sum_{m=0}^{\infty} \sum_{q_{1}}^{+j}(\ldots) \\
g_{-}(X) & =\sum_{j=0}^{\infty} \sum_{m=-\infty}^{-2 j} \sum_{q_{1} q_{2}=-j}^{+j}(\ldots) \\
g_{0}(X) & =\sum_{j=1}^{\infty} \sum_{m=-2} \sum_{j+1}^{+1}(\ldots) .
\end{aligned}
$$

They are all elements of the space $\mathscr{L}^{2}(\$)$. From (1.72) and (1.73) follows

$$
g_{+}(X)+g_{-}(X)+g_{0}(X)=g(X)+a_{00}^{00} .
$$

We treat the positive part first. We make use of

$$
e^{i(m+j) \varphi} D_{q_{1} q_{2}}^{j}(u)=(\operatorname{det} X)^{m} D_{q_{1} q_{2}}^{j}(X) .
$$

If we let the real parameters $\alpha, \beta, \gamma, \delta(1.69)$ and $\varphi(1.67)$ assume complex values, $X$ appears as the boundary value of $Z$ and $(\operatorname{det} X)^{m} D_{q_{1} q_{2}}^{j}(X)$ as the boundary value of $(\operatorname{det} Z)^{m} D_{q_{1} q_{2}}^{j}(Z)$. In a sense still to be specified we may therefore consider the series

$$
f_{+}(Z)=\sum_{m=0}^{\infty} \sum_{j=0}^{\infty} \sum_{q_{1} q_{2}=-j}^{+j} a_{q_{1} q_{2}}^{j m}(2 j+1)^{\frac{1}{2}} N^{j m} \Delta_{q_{1} q_{2}}^{j m}(Z)
$$

as the holomorphic extension of $g_{+}(X)$ into $\mathbb{D}$.

It is easy to see that the coefficients $(2 j+1)^{\frac{1}{2}} N^{j m}$ are bounded for any fixed $n \geqq 2$. Parseval's equation for the part $g_{+}(X)$ in $\mathscr{L}^{2}(\mathbb{S})$ yields

$$
\left\|g_{+}\right\|_{\mathscr{L}^{2}(\$)}^{2}=\int\left|g_{+}(X)\right|^{2} d \mu(X)=\sum_{m=0}^{\infty} \sum_{j=0}^{\infty} \sum_{q_{1} q_{2}=-j}^{+j}\left|a_{q_{1} q_{2}}^{j m}\right|^{2} .
$$

Consequently $f_{+}(Z)$ converges in the norm of $\mathscr{H}_{n}(\mathbb{D})$ for all $n \geqq 2$, that means in particular, it converges uniformly in each compact subset ofID towards a holomorphic function. This holomorphic function assumes the boundary value $g_{+}(X)$ on the Shilov boundary $\mathbb{S}$ in the sense of an $\mathscr{L}^{2}(\mathbb{S})$ limit.

The negative part can be treated quite analogously. We have [3]

$$
\begin{aligned}
e^{i(m+j) \varphi} D_{q_{1} q_{2}}^{j}(u) & =(-1)^{q_{1}-q_{2}} e^{i(m+j) \varphi} D_{-q_{2},-q_{1}}^{j}\left(u^{+}\right) \\
& =(-1)^{q_{1}-q_{2}} e^{i(m+2 j) \varphi} D_{-q_{2},-q_{1}}^{j}\left(X^{+}\right) \\
& =(-1)^{q_{1}-q_{2}}\left(\operatorname{det} X^{+}\right)^{-m-2 j} D_{-q_{2},-q_{1}}^{j}\left(X^{+}\right) .
\end{aligned}
$$


We introduce the shorthands

$$
\begin{gathered}
m^{\prime}=-m-2 j, \quad m^{\prime}=0,1,2, \ldots \\
\tilde{a}_{q_{1} q_{2}}^{j m^{\prime}}=(-1)^{q_{1}-q_{2}} a_{-q_{2},-q_{1}}^{j m}
\end{gathered}
$$

and get as antiholomorphic extension of $g_{-}(X)$

$$
f_{-}(Z)=\sum_{m^{\prime}=0}^{\infty} \sum_{j=0}^{\infty} \sum_{q_{1} q_{2}=-j}^{+j} \tilde{a}_{q_{1} q_{2}}^{j m^{\prime}}(2 j+1)^{\frac{1}{2}} N^{j m^{\prime}} \Delta_{q_{1} q_{2}}^{j m^{\prime}}\left(Z^{+}\right) .
$$

This series is uniformly convergent on any compact subset of $\mathbb{D}$ towards an antiholomorphic function, it lies in $\mathscr{H}_{n}^{*}(\mathrm{DD})$ for all $n \geqq 2$, and assumes the function $g_{-}(X)$ on the Shilov boundary.

For the sake of brevity we denote the subspaces of functions $g_{+}(X)$ and $g_{-}(X)$ by $\mathscr{L}_{+}^{2}(\mathbb{S})$ and $\mathscr{L}_{-}^{2}(\mathbb{S})$, respectively. An elegant presentation of the mapping of $\mathscr{L}_{+}^{2}(\mathbb{S})$ into $\mathscr{H}_{n}(\mathbb{D})\left(\right.$ of $\mathscr{L}_{-}^{2}(\mathbb{S})$ into $\left.\mathscr{H}_{n}^{*}(\mathbb{D})\right)$ can be given by means of the Szegö kernel function $K^{S}\left(Z_{1}, Z_{2}\right)[4]$.

Let $\hat{\mathscr{H}}_{n}(\mathrm{D})$ denote the image of $\mathscr{L}_{+}^{2}(\mathbb{S})$ under the extension $g_{+}(X) \rightarrow f_{+}(Z)$. In this subspace we introduce the Szegö norm

$$
\left\|f_{+}\right\|_{S}=\left\|g_{+}\right\|_{\mathscr{L}^{2}(\$)}
$$

and a corresponding scalar product. A Szegö orthonormal basis in $\hat{\mathscr{H}}_{n}(\mathrm{DD})$ is then given by

$$
\begin{gathered}
S_{q_{1} q_{2}}^{j m}(Z)=(2 j+1)^{\frac{1}{2}}(\operatorname{det} Z)^{m} D_{q_{1} q_{2}}^{j}(Z) \\
m=0,1,2, \ldots, \quad-j \leqq q_{1}, q_{2} \leqq+j, \quad 2 j=0,1,2, \ldots
\end{gathered}
$$

The Szegö kernel function is then defined by

$$
K^{S}\left(Z_{1}, Z_{2}\right)=\sum_{m=0}^{\infty} \sum_{j=0}^{\infty} \sum_{q_{1} q_{2}=-j}^{+j} S_{q_{1} q_{2}}^{j m}\left(Z_{1}\right) \overline{S_{q_{1} q_{2}}^{j m}\left(Z_{2}\right)}
$$

Comparing (1.82) with (1.48), (1.49) we recognize that the Szegö kernel is identical with the Bergman kernel for $n=2$. Therefore from(1.64) we have

$$
K^{S}\left(Z_{1}, Z_{2}\right)=\left[\operatorname{det}\left(E-Z_{1} Z_{2}^{+}\right)\right]^{-2} .
$$

If $Z_{1} \in \mathbb{D}$ but $Z_{2}=X$ lies on the Shilov boundary, the Szegö kernel function is holomorphic in $Z_{1}$ and square integrable in $X$ with respect to the measure (1.68). We denote

and have

$$
K_{Z}^{S}(X)=K^{S}(Z, X)
$$

respectively

$$
\begin{gathered}
f_{+}(Z)=\left(\overline{K_{Z}^{S}}, g\right)_{\mathscr{L}^{2}(\$)}=\left(\overline{K_{Z}^{S}}, g_{+}\right)_{\mathscr{L}^{2}(\$)} \\
f_{-}(Z)=\left(K_{Z}^{S}, g\right)_{\mathscr{L}^{2}(\$)}=\left(K_{Z}^{S}, g_{-}\right)_{\mathscr{L}^{2}(\$)} .
\end{gathered}
$$

5 Commun math Phys., Vol. 27 
The formulae (1.86) and (1.87) can be viewed upon as generalizations of Hilbert transforms.

One can make use of the integral representation (1.86) in the following manner that will become important in the sequel. From (1.86) and the hermiticity of the Szegö kernel we deduce by means of Schwarz's inequality

$$
\begin{aligned}
\left|f_{+}(Z)\right| & \leqq\left\|K_{Z}^{S}\right\|_{\mathscr{L}^{2}(\$)}\left\|g_{+}\right\|_{\mathscr{L}^{2}(\$)} \\
& =\left\|g_{+}\right\|_{\mathscr{L}^{2}(\$)} K^{S}(Z, Z)^{\frac{1}{2}}
\end{aligned}
$$

Inserted into (1.26) we obtain an estimate for the norm of $f_{+}(Z)$

$$
\left\|f_{+}\right\|_{n}^{2} \leqq c\left\|g_{+}\right\|_{\mathscr{L}^{2}(\$)}^{2} \int_{\mathbb{j})}\left[\operatorname{det}\left(E-Z^{+} Z\right)\right]^{n-6}|d Z| .
$$

This norm is therefore finite whenever $n \geqq 6$. This estimate is therefore less restrictive than the one found by estimating the coefficients of the Fourier series, which resulted in a finite norm for all $n \geqq 2$.

\subsection{Distributions on the Shilov Boundary}

We consider the space of infinitely differentiable functions on $\mathbb{S}$ with the usual topology which we denote by $\mathscr{E}(\mathbb{S})$. The continuous linear functionals on this space form the dual space $\mathscr{E}^{\prime}(\mathbb{S})$ of distributions on $\mathbb{\$}$. Each distribution $\varphi(X)$ can be expanded in a generalized Fourier series like the functions of $\mathscr{L}^{2}(\$)$ but the expansion coefficients are now allowed to increase polynomially (see (1.72))

$$
\sum_{q_{1} q_{2}=-j}^{+J}\left|a_{q_{1} q_{2}}^{j m}\right|^{2} \leqq C(1+|m|+j)^{s}
$$

rather than being square summable. $s$ is a fixed integer, $C$ any positive constant depending only on $\varphi$.

Such Fourier expansion allows us to split each distribution $\varphi(X)$ uniquely into three parts $\varphi_{+}(X), \varphi_{-}(X), \varphi_{0}(X)$ just as in (1.73). These parts add up to

$$
\varphi_{+}(X)+\varphi_{-}(X)+\varphi_{0}(X)=\varphi(X)+a_{00}^{00} .
$$

All these Fourier expansions of distributions converge in the topology of $\mathscr{E}^{\prime}(\mathbb{S})$. Another way to define the parts of $\varphi(X)$ is by requiring

$$
\int \varphi_{ \pm}(X) \overline{g(X)} d \mu(X)=\int \varphi(X) \overline{g_{ \pm}(X)} d \mu(X)
$$

for any test function $g(X)$.

We consider the Szegö kernel (1.84) with the first argument $Z$ in $\mathbb{D}$ and the second argument $X$ on $\mathbb{S}$. Then it is infinitely differentiable in 
$X$ for fixed $Z$. Therefore we can define Hilbert transforms of the distribution $\varphi(X)$ (where the notation $(\ldots, \ldots)$ denotes the scalar product of $\mathscr{L}^{2}(\mathbb{S})$ extended to test functions and distributions)

$$
\begin{aligned}
& f_{+}(Z)=\left(\overline{K_{Z}^{S}}, \varphi\right)=\left(\overline{K_{Z}^{S}}, \varphi_{+}\right), \\
& f_{-}(Z)=\left(K_{Z}^{S}, \varphi\right)=\left(K_{Z}^{S}, \varphi_{-}\right) .
\end{aligned}
$$

These are holomorphic, respectively antiholomorphic in $\mathbb{D}$. In fact we shall see that they lie in the Hilbert spaces $\mathscr{H}_{n}(\mathbb{D})$ or $\mathscr{H}_{n}^{*}(\mathbb{D})$ whenever $n \geqq n_{0}$. We construct a connection between $n_{0}$ on the one hand and the polynomial order $s$ in (1.90) on the other hand. However, we start formulating another theorem first.

Let $f(Z)$ be an element of $\mathscr{H}_{n}(\mathbb{D})$. Then $f(Z)$ approaches a distribution $\varphi(X) \in \mathscr{E}^{\prime}(\mathbb{S})$ if $Z$ tends to $X \in \mathbb{S}$ in the sense of the topology of $\mathscr{E}^{\prime}(\mathbb{S}) . \varphi(X)$ can be represented as the $(n-2) n d$ derivative of a square integrable function $g_{+}(X) \in \mathscr{L}_{+}^{2}(\mathbb{S})$. In turn, the holomorphic extension (1.93) of $\varphi(X)$ gives us back $f(Z)$. (Theorem A).

For the proof we start from the expansion

$$
f(Z)=\sum_{m=0}^{\infty} \sum_{j=0}^{\infty} \sum_{q_{1} q_{2}=-j}^{+j} a_{q_{1} q_{2}}^{j m} \Delta_{q_{1} q_{2}}^{j m}(Z)
$$

The basis elements $\Delta_{q_{1} q_{2}}^{j m}(Z)$ are homogeneous polynomials in the elements of $Z$ of degree $N=2 j+2 m$. Therefore Euler's differential operator yields

$$
\sum_{i j} z_{i j} \frac{\partial}{\partial z_{i j}} \Delta_{q_{1} q_{2}}^{j m}(Z)=N \Delta_{q_{1} q_{2}}^{j m}(Z) .
$$

Hence $f(Z)$ can be represented in the form

$$
f(Z)=\left(\sum_{l j} z_{i j} \frac{\partial}{\partial z_{i j}}+1\right)^{n-2} \sum_{m . j, q_{1}, q_{2}} b_{q_{1} q_{2}}^{j m}(2 j+1)^{\frac{1}{2}}(\operatorname{det} Z)^{m} D_{q_{1} q_{2}}^{j}(Z)
$$

where

$$
b_{q_{1} q_{2}}^{j m}=\left\{(2 j+1)^{\frac{1}{2}} N^{j m}(2 j+2 m+1)^{n-2}\right\}^{-1} a_{q_{1} q_{2}}^{j m} .
$$

It is an easy task to prove that the factor in curly brackets in (1.98) increases both with $j$ and $m$ monotonically such that its minimum is assumed for $j=m=0$. This minimum is one. Consequently

$$
\left|b_{q_{1} q_{2}}^{j m}\right| \leqq\left|a_{q_{1} q_{2}}^{j m}\right|
$$

and by Parseval's equation

$$
\sum_{m, j, q_{1} q_{2}}\left|b_{q_{1} q_{2}}^{j m}\right|^{2} \leqq\|f\|_{n}^{2}<\infty .
$$


Therefore we can write

where

$$
f(Z)=\left(\sum_{i j} z_{i j} \frac{\partial}{\partial z_{\imath j}}+1\right)^{n-2} h(Z)
$$

$$
h(Z) \rightarrow g_{+}(X) \in \mathscr{L}_{+}^{2}(\mathbb{S}) .
$$

The differential operator in (1.101) tends towards a differential operator of order $n-2$ on $\mathbb{\$}$. This completes the proof.

We consider next a distribution

$$
\varphi(X)=\sum_{m=0}^{\infty} \sum_{j=0}^{\infty} \sum_{q_{1} q_{2}=-j}^{+j} a_{q_{1} q_{2}}^{j m}(2 j+1)^{\frac{1}{2}}(\operatorname{det} X)^{m} D_{q_{1} q_{2}}^{j}(X)
$$

so that $\varphi(X)$ is equal to its positive part. We define an "integral order" $n_{0}$ of $\varphi(X)$. For this purpose we introduce the notation

$$
\sigma_{N}=\sum_{\substack{j, m \\ N \text { fixed }}} \sum_{q_{1} q_{2}=-j}^{+j}\left|a_{q_{1} q_{2}}^{j m}\right|^{2}, \quad N=2 j+2 m .
$$

Due to $(1.90)$ there exist real numbers $\omega$ such that

$$
\sum_{N=0}^{\infty} \sigma_{N}(1+N)^{-2 \omega}<\infty
$$

Let $n_{0}$ be the smallest integer in the set of these $\omega$ (the possibility $n_{0}=-\infty$ is admitted). Then we can represent $\varphi(X)$ in the form

$$
\begin{aligned}
\varphi(X)= & \left(\sum_{i j} x_{i j} \frac{\partial}{\partial x_{i j}}+1\right)^{n_{0}^{\prime}} \sum_{m, j, q_{1} q_{2}}(N+1)^{-n_{0}^{\prime}} a_{q_{1} q_{2}}^{j m} \\
& \cdot(2 j+1)^{\frac{1}{2}}(\operatorname{det} X)^{m} D_{q_{1} q_{2}}^{j}(X), \quad n_{0}^{\prime}=\max \left(n_{0}, 0\right) .
\end{aligned}
$$

Hence due to (1.105) it appears as the $n_{0}^{\prime}-t h$ order derivative of a square integrable function $g_{+}(X) \in \mathscr{L}_{+}^{2}(\mathbb{S})$.

Let $n_{0}$ be the integral order of the distribution $\varphi(X), \varphi(X)=\varphi_{+}(X)$. Then $\varphi(X)$ possesses a holomorphic extension of the type (1.93) that lies in $\mathscr{H}_{n}(\mathbb{D})$ for all

$$
n \geqq 2 n_{0}^{\prime}+2, \quad n_{0}^{\prime}=\max \left(n_{0}, 0\right) .
$$

With the help of the representation (1.106) this extension can also be written as

$$
F(Z)=\left(\sum_{i j} z_{i j} \frac{\partial}{\partial z_{i j}}+1\right)^{n_{0}^{\prime}} f_{+}(Z)
$$

where $f_{+}(Z)$ is the extension of $g_{+}(X) \in \mathscr{L}_{+}^{2}(\mathbb{S})$ (Theorem B).

In order to prove this theorem we must only show that the premise

$$
\sum_{N=0}^{\infty} \sigma_{N}(N+1)^{-2 n_{0}^{\prime}}<\infty
$$


implies

$$
\sum_{m=0}^{\infty} \sum_{j=0}^{\infty} \sum_{q_{1} q_{2}=-j}^{+j}\left|b_{q_{1} q_{2}}^{j m}\right|^{2}<\infty
$$

for all $n \geqq 2 n_{0}^{\prime}+2$. Here $b_{q_{1} q_{2}}^{j m}$ denotes

$$
b_{q_{1} q_{2}}^{j m}=(2 j+1)^{\frac{1}{2}} N^{j m} a_{q_{1} q_{2}}^{j m} .
$$

In fact, we get

$$
\begin{aligned}
\sum_{\substack{j, m \\
N \text { fixed }}} \sum_{q_{1} q_{2}=-j}^{+j}\left|b_{q_{1} q_{2}}^{j m}\right|^{2} & \leqq \sigma_{N}\left\{\max _{N \text { fixed }}(2 j+1)\left(N^{j m}\right)^{2}\right\} \\
& =\frac{(n-1) !(N+1) !(N+1)^{2 n_{0}^{\prime}}}{(N+n-1) !}\left\{\sigma_{N}(N+1)^{-2 n_{0}^{\prime}}\right\} .
\end{aligned}
$$

For all $n$ satisfying (1.107) the factor in front of the curly bracket is bounded in $N$ by a number $C_{n}$. Therefore (1.110) follows.

Another version of Theorem B is obtained with the help of the Szegö kernel. We know from the general theory of distributions [8] that a distribution $\varphi(X) \in \mathscr{E}^{\prime}(\mathbb{S})$ can be represented as

$$
\varphi(X)=D_{X}^{m} g(X)
$$

where $D_{X}^{m}$ is a differential operator of order $|m|,|m|=\sum_{i j} m_{i j}$,

$$
D_{X}^{m}=\sum_{l \leqq m} p_{l}(X) \prod_{i j}\left(\frac{\partial}{\partial x_{l j}}\right)^{l_{l j}}, \quad p_{l}(X) \text { are polynomials }
$$

and $g(X)$ is square integrable. The holomorphic extension of $\varphi_{+}(X)$ is obviously

$$
f_{+}(Z)=D_{Z}^{m} \int_{\Phi} K^{S}(Z, X) g_{+}(X) d \mu(X)
$$

Schwarz's inequality implies

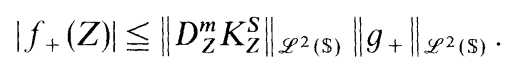

The hermiticity of the Szegö kernel and (1.86) yield

$$
\begin{aligned}
\left\|D_{Z}^{m} K_{Z}^{S}\right\|_{\mathscr{L}^{2}(\$)}^{2} & =D_{Z}^{m} D_{Z}^{m} K^{S}(Z, Z) \\
& \leqq M\left[\operatorname{det}\left(E-Z Z^{+}\right)\right]^{-2-2|m|}
\end{aligned}
$$

Inserting the last two expressions into the norm (1.26) gives

$$
\left\|f_{+}\right\|_{n}^{2} \leqq c M\left\|g_{+}\right\|_{\mathscr{L}^{2}(\$)}^{2} \int_{\mathbb{D}}\left[\operatorname{det}\left(E-Z^{+} Z\right)\right]^{n-6-2|m|}|d Z|
$$

which is finite for $n \geqq 2|m|+6$. If we use the representation (1.106) we find $n_{0}^{\prime}=|m|$, and we have finally the condition $n \geqq 2 n_{0}^{\prime}+6$ instead of (1.107). 


\subsection{The Delta Distribution}

As an example we study the delta distribution on $\mathbb{S}$ that is defined by

$$
\int_{\Phi} g(X) \delta(X) d \mu(X)=g(E)
$$

for all continuous functions $g(X)$ on $\mathbb{\$}$. In the expansion

$$
\delta(X)=\sum_{m=-\infty}^{+\infty} \sum_{j=0}^{\infty} \sum_{q_{1} q_{2}=-j}^{+j} a_{q_{1} q_{2}}^{j m}(2 j+1)^{\frac{1}{2}}(\operatorname{det} X)^{m} D_{q_{1} q_{2}}^{j}(X)
$$

the coefficients are easily computed from (1.119)

This yields

$$
a_{q_{1} q_{2}}^{j m}=(2 j+1)^{\frac{1}{2}} \delta_{q_{1} q_{2}} .
$$

$$
\delta_{+}(X)=\sum_{m=0}^{\infty} \sum_{j=0}^{\infty}(2 j+1)(\operatorname{det} X)^{m} \operatorname{Tr} D^{j}(X) .
$$

The positive part of the delta distribution possesses the analytic extension

$$
d_{+}(Z)=K^{S}(Z, E)=[\operatorname{det}(E-Z)]^{-2}
$$

as can be seen most easily from (1.93). Similarly the distribution $\delta_{-}(X)$ possesses the antiholomorphic extension

$$
d_{-}(Z)=K^{S}(E, Z)
$$

The norm of $d_{+}(Z)$ in $\mathscr{H}_{n}(\mathbb{D})$ can be computed explicitly. After some algebra we find

$$
\left\|d_{+}\right\|_{n}^{2}=\frac{(n-1)(n-2)^{2}}{(n-4)^{2}(n-5)} .
$$

It follows that $d_{+}(Z)$ lies in all $\mathscr{H}_{n}(\mathbb{D})$ for $n \geqq 6$.

This exact result can be compared with the proposition of Theorem B. From (1.46) we have

$$
\sigma_{N}=S_{N}=\frac{1}{6}(N+1)(N+2)(N+3)
$$

so that $\omega$ is any real number bigger than two. The integral order $n_{0}$ is three. From (1.107) we have $n \geqq 8$. On the other hand the property that $n_{0}$ is an integer enters only the second part of Theorem B. The assertion of the first part can be improved if we allow also for half-integral $n_{0}$. Let us denote these "half-integral order" $n_{00}$. In the present example we find $n_{00}=5 / 2$ and from (1.107) the stronger estimate $n \geqq 7$. 


\section{The Coset Space as a Tube Domain in Complex Minkowski Space}

\subsection{Preliminary Remarks}

We define the complex Minkowski space $\mathbb{C}_{4}$ as the vector space of complex four vectors

$$
w=\left(w_{0}, w_{1}, w_{2}, w_{3}\right) \text {. }
$$

Real four vectors constitute the real Minkowski space $\mathbb{M}_{4}$. Both in $\mathbb{C}_{4}$ and in $\mathbb{M}_{4}$ we have a bilinear form

$$
w w^{\prime}=w_{0} w_{0}^{\prime}-\sum_{k=1}^{3} w_{k} w_{k}^{\prime}, \quad w w=w^{2} .
$$

In $\mathbb{C}_{4}$ we define a tube domain by

$$
\mathrm{T}=\left\{w \mid w=u+i v, u, v \in \mathbb{M}_{4}, v_{0}>\left(\sum_{i=1}^{3} v_{i}^{2}\right)^{\frac{1}{2}}\right\}
$$

which we call the tube domain in the sequel.

The tube domain plays an important role in field theory. We define the forward light cone IL in $\mathbb{M}_{4}$ by

$$
\mathbb{L}=\left\{u \mid u_{0}>\left(\sum_{i=1}^{3} u_{i}^{2}\right)^{\frac{1}{2}}\right\}
$$

Then the Fourier transform of any tempered distribution of $\mathscr{S}^{\prime}\left(\mathbb{M}_{4}\right)$ with support in $\mathbb{L}$ (by a bar over a set we mean the closure) is itself a tempered distribution which possesses a holomorphic extension into $\mathbb{T}$. In turn this holomorphic function assumes the Fourier transform on the boundary $\mathbb{M}_{4}$ of $\mathbb{T}$ in the sense of a limit in the $\mathscr{S}^{\prime}\left(\mathbb{M}_{4}\right)$ topology [9].

\subsection{A Transformation of the Cayley Type}

We introduce a mapping of the bounded manifold $\mathbb{D}$ into $\mathbb{C}_{4}$ by

$$
\begin{aligned}
& W=W(Z)=i(E-Z)(E+Z)^{-1} \\
& W=w_{0} E+\sum_{i=1}^{3} w_{i} \sigma_{i}
\end{aligned}
$$

$\sigma_{i}$ are the Pauli matrices. If $Z$ is unitary, $W$ is hermitean and vice versa. The definition (1.20) of $\mathbb{D}$ guarantees that $W=W(Z)$ is holomorphic on $\mathbb{D}$. The inverse mapping is

$$
Z=Z(W)=(E-i W)^{-1}(E+i W) .
$$

Inserting (2.6) into (1.20) yields

$$
\left(E+i W^{+}\right)\left(E-Z^{+} Z\right)(E-i W)=2 i\left(W^{+}-W\right) .
$$


If we put $W=U+i V, w=u+i v, u, v \in \mathbb{M}_{4}$, we have from (2.7) that $V$ is positive semi-definite (it turns out to be positive definite, indeed) for all $Z \in \mathbb{D}$. This means

$$
\operatorname{Sp} V=2 v_{0} \geqq 0, \quad \operatorname{det} V=v^{2} \geqq 0
$$

or $w \in \overline{\mathbb{T}}$.

In turn, if $w \in \overline{\mathbb{T}}$, then $\operatorname{det}(E-i W) \neq 0$. To show this we set $w=u+i v$, $v \in \overline{\mathbb{L}}$, and get

$$
\operatorname{det}(E-i W)=\left(1+2 v_{0}-u^{2}+v^{2}\right)-2 i\left(u_{0}+u v\right) .
$$

Therefore, if $\operatorname{det}(E-i W)=0$, then

$$
u^{2}=1+2 v_{0}+v^{2} \geqq 1
$$

since $v \in \overline{\mathbb{L}}$. Consequently we have $u_{0} \neq 0$ and $\operatorname{sign} u_{0}=\operatorname{sign}(u v)$ whenever $v \neq 0$. In any case it follows $u_{0}+u v \neq 0$ for all $v \in \overline{\mathbb{L}}$. This contradicts $\operatorname{det}(E-i W)=0$.

It follows that the mapping $W=W(Z)$ is one-to-one and pseudoconformal from $\mathbb{D}$ onto $\mathbb{T}$.

We denote those points of the boundary $\hat{c} \mathbb{D}$ where one of the two eigenvalues of $Z$ is equal to -1 by $\mathbb{N}$. On $\partial \mathbb{D}-\mathbb{N}$ the mapping (2.5) is continuous and the image is the boundary of $\mathbb{T}$. If in addition $Z$ is unitary, namely an element of the Shilov boundary $\mathbb{S}$, then $W$ is hermitean and the set $\mathbb{S}-(\mathbb{S} \cap \mathbb{N})$ is consequently mapped one-to-one on $\mathbb{M}_{4}$.

The Lebesgue measure $|d Z|$ on $\mathbb{D}(1.24)$ and the Lebesgue measure on $\mathbb{T}$

$$
|d W|=\prod_{\mu=0}^{3} d \operatorname{Re} w_{\mu} d \operatorname{Im} w_{\mu}=d^{4} u d^{4} v
$$

are connected by the functional determinant $J_{1}$

$$
|d Z|=J_{1}|d W|
$$

where elementary algebra yields

$$
J_{1}=2^{-4}|\operatorname{det}(E+Z)|^{8}=2^{12}|\operatorname{det}(E-i W)|^{-8} .
$$

Similarly the measure $d \mu(X)$ on $\$(1.68)$ and the Lebesgue measure on $\mathbb{M}_{4}$ are related by the functional determinant $J_{2}$

with

$$
d \mu(X)=J_{2} d^{4} u
$$

$$
J_{2}=\left(\frac{2}{\pi}\right)^{3}|\operatorname{det}(E-i U)|^{4} \text {. }
$$




\subsection{The Tube Domain as a Homogeneous Space}

The pseudo-conformal mapping (2.5) induces a transformation of $\mathbb{T}$ by means of the rational transformation (1.23) of $\mathbb{D}$. This transformation of $T$ is again rational and allows the ansatz

$$
W^{\prime}=(R W+S)(T W+Q)^{-1} .
$$

Inserting (2.5) into (2.15), solving for $Z^{\prime}$, and comparing with (1.23) we get

$$
\begin{aligned}
& A=\frac{1}{2}(R+i S-i T+Q) \\
& B=\frac{1}{2}(-R+i S+i T+Q) \\
& C=\frac{1}{2}(-R-i S-i T+Q) \\
& D=\frac{1}{2}(R-i S+i T+Q)
\end{aligned}
$$

where we fixed an overall constant factor for convenience. The question arises as to how the set of constraints (1.4) on $A, B, C, D$ that guarantee that the matrix $M$ lies in $U(2,2)$, maps on an equivalent set of constraints for $R, S, T, Q$. We give the result without the lengthy derivation. The set desired is

$$
\begin{aligned}
R^{+} T & =H_{1} \\
R^{+} Q & =E+H_{2}-i H_{3} \\
S^{+} T & =H_{2}+i H_{3} \\
S^{+} Q & =H_{4}
\end{aligned}
$$

where $H_{i}, i=1$ to 4 , are hermitean but otherwise arbitrary matrices.

We consider two subgroups of $S U(2,2)$ in detail. We make use of the fact that the transformations (2.15) themselves have the group structure $S U(2,2) / Z_{4}$ (where $Z_{4}$ denotes the four element centre). The first subgroup $G_{1}$ is defined by the premise $T=0$. That this constraint defines a subgroup will turn out immediately (see (2.19)). From (2.17) follows

$$
\begin{aligned}
H_{1} & =H_{2}=H_{3}=0 \\
R^{+} & =Q^{-1} \\
S & =R H_{4} .
\end{aligned}
$$

Thus (2.15) reduces to

$$
W^{\prime}=(R W+S) Q^{-1}=R\left(W+H_{4}\right) R^{+}
$$

If $\operatorname{det} R=1$, this transformation (2.19) consists of a real translation and a real pseudo-rotation in $\mathbb{T}$. This subgroup of $G_{1}$ defined by the constraint $\operatorname{det} R=1$ is identical with the "inhomogeneous $S L(2, C)$ " 
group. However, $\operatorname{det} R$ need not be equal to one. Let us assume

Then

$$
\begin{aligned}
& R=\lambda E, \quad \lambda=e^{\eta_{1}+i \eta_{2}}, \quad \eta_{1,2} \text { real } \\
& S=H_{4}=0 .
\end{aligned}
$$

$$
W^{\prime}=e^{2 \eta_{1}} W .
$$

Due to (2.16) we have

$$
A=D=e^{i \eta_{2}} \operatorname{ch} \eta_{1} E, \quad B=C=-e^{i \eta_{2}} \operatorname{sh} \eta_{1} E
$$

so that

$$
\left(\begin{array}{ll}
A & B \\
C & D
\end{array}\right)=e^{i \eta_{2}}\left(\begin{array}{rr}
\operatorname{ch} \eta_{1} E, & -\operatorname{sh} \eta_{1} E \\
-\operatorname{sh} \eta_{1} E, & \operatorname{ch} \eta_{1} E
\end{array}\right)
$$

with the determinant $e^{4 i \eta_{2}}$. In order that this matrix is in $S U(2,2)$ we must have

$$
e^{i \eta_{2}} \in\{1,+i,-1,-i\} .
$$

If $\eta_{1}=0$ the elements (2.20) constitute the central subgroup $Z_{4}$ of $S U(2,2)$ with the four elements (2.24). If $\eta_{2}=0$ the elements $(2.20)$ form a one-parameter subgroup of dilations. We denote it $D$. Then $G_{1}$ has the total content

$$
G_{1}=\left\{D \times\left[S L(2, C) / Z_{2} \times T_{4}\right]\right\} \otimes Z_{4}
$$

where $T_{4}$ is the group of real translations in $\mathbb{T}$ and $\times$ denotes the semidirect product.

The other subgroup of $S U(2,2)$ that is of particular interest, $G_{2}$, is defined by the requirements

From (2.17) follows

$$
S=0, \quad Q=E .
$$

$$
\begin{aligned}
H_{2} & =H_{3}=H_{4}=0 \\
R & =E \\
T & =H_{1}=t_{0} E-\sum_{k=1}^{3} t_{k} \sigma_{k}, t_{\mu} \text { real } .
\end{aligned}
$$

The transformation (2.15) reduces in this case to

Since

$$
W^{\prime}=W(E+T W)^{-1} .
$$

$$
\operatorname{det}(E+T W)=1+2 t w+t^{2} w^{2}
$$

we get from (2.28)

$$
w_{\mu}^{\prime}=\frac{w_{\mu}+w^{2} t_{\mu}}{1+2 t w+t^{2} w^{2}} .
$$


These are the "special conformal transformations" of $T$. They form an abelian four-dimensional Lie group. $G_{2}$ is free of central elements of $S U(2,2)$.

\subsection{Hilbert Spaces}

In order to avoid too many repetitions we discuss only spaces of holomorphic functions from now on. The spaces of antiholomorphic functions can be treated analogously.

We start from the spaces $\mathscr{H}_{n}(\mathbb{D})$. Let $f(Z)$ be any function of $\mathscr{H}_{n}(\mathbb{D})$. Then we define a function $F(w)$ which is holomorphic in $\mathbb{T}$ by

$$
F(w)=m_{q}(w) f(Z(w))
$$

where $m_{q}(w)$ is a multiplier function that has to be holomorphic in $\mathbb{T}$, too. For later purposes the following class of multipliers is most convenient

$$
m_{q}(w)=2^{2 n-2}[\operatorname{det}(E-i W)]^{-n+q}
$$

where $q$ is any integer.

For two functions $F_{1,2}(w)$ defined by (2.31) with the same $q$ we introduce the scalar product (see (1.49) and (1.52))

$$
\left(F_{1}, F_{2}\right)_{n, q}=c \int_{\mathrm{T}} \overline{F_{1}(w)} F_{2}(w)\left[(\operatorname{Im} w)^{2}\right]^{n-4}|[\operatorname{det}(E-i W)]|^{-2 q}|d W|
$$

such that

$$
\left(F_{1}, F_{2}\right)_{n, q}=\left(f_{1}, f_{2}\right)_{n} .
$$

Both (2.31) and (2.33) establish a natural isomorphism of the space $\mathscr{H}_{n}(\mathbb{D})$ on a Hilbert space $\mathscr{H}_{n, q}(\mathbb{T})$ of holomorphic functions on $\mathbb{T}$.

By means of the pseudo-conformal mapping (2.5) $\mathbb{T}$ has become a homogeneous space for $U(2,2)$. We assume that $A, B, C, D$ and correspondingly $R, S, T, Q$ belong to the matrix $M^{-1}$. Then we can read off the mapping $F \rightarrow T_{M} F$ from the diagram

It has the form

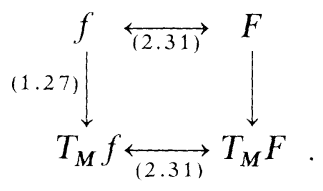

$$
T_{M} F(w)=\mu_{q}(M, w) F\left(w^{\prime}\right)
$$

with $w^{\prime}$ defined by $(2.15)$ and a multiplier $\mu_{q}(M, w)$ that can be computed from (2.31) and (2.32). We find

$$
\begin{aligned}
\mu_{q}(M, w)= & {\left[\operatorname{det}(E-i W)\left(E-i W^{\prime}\right)^{-1}\right]^{q} } \\
& {[\operatorname{det}(T W+Q)]^{-n} . }
\end{aligned}
$$


The case $q=0$ is of particular simplicity both in (2.33) and (2.36). Nevertheless, we need also the cases $q \neq 0$. The operators $T_{M}$ present another realization of the same unitary representations of $S U(2,2)$ that were defined on the function spaces $\mathscr{H}_{n}(\mathbb{D})$.

The Bergman kernel function for the Hilbert spaces $\mathscr{H}_{n, q}(\mathbb{T})$ is easily computed. We require

$$
F(w)=\left(\overline{K_{w}^{B}}, F\right)_{n, q}
$$

for all $F \in \mathscr{H}_{n, q}(T)$. With the Bergman kernel (1.64) for the spaces $\mathscr{H}_{n}(\mathbb{D})$ and the mapping (2.31) we obtain

$$
\begin{aligned}
K_{w_{1}}^{B}\left(w_{2}\right) & =K^{B}\left(w_{1}, w_{2}\right) \\
& =m_{q}\left(w_{1}\right) \overline{m_{q}\left(w_{2}\right)} K^{B}\left(Z\left(w_{1}\right), Z\left(w_{2}\right)\right) .
\end{aligned}
$$

Inserting (2.6) and (2.32) yields

$$
\begin{aligned}
K^{B}\left(w_{1}, w_{2}\right)= & 2^{-4}\left[\operatorname{det}\left(E-i W_{1}\right)\left(E+i W_{2}^{+}\right)\right]^{q} \\
& \cdot\left\{\operatorname{det}\left[-\frac{i}{2}\left(W_{1}-W_{2}^{+}\right)\right]\right\}^{-n}
\end{aligned}
$$

By means of Schwarz's inequality we obtain from (2.37) for any $F \in \mathscr{H}_{n, q}(\mathbb{T})$

$$
\begin{aligned}
F(w) & \leqq\|F\|_{n, q} K^{B}(w, w)^{\frac{1}{2}} \\
& =\frac{1}{4}\|F\|_{n, q}|\operatorname{det}(E-i W)|^{q}[\operatorname{det}(\operatorname{Im} W)]^{-\frac{n}{2}} .
\end{aligned}
$$

The label $q$ has obviously been introduced to account for the polynomial increase of tempered distributions at infinity.

Similarly we consider the mapping of $\mathbb{S}$ on $\mathbb{M}_{4}$ induced by the mapping (2.5). Remembering the relation (2.13), (2.14) between the respective measures, we define

and

$$
\begin{aligned}
G(u) & =[\operatorname{det}(E-i U)]^{-2} g(X(u)) \\
U & =u_{0} E+\sum_{k=1}^{3} u_{k} \sigma_{k}, u_{\mu} \in \mathbb{M}_{4}
\end{aligned}
$$

$$
\begin{aligned}
\left(G_{1}, G_{2}\right)_{\mathscr{L}^{2}\left(\mathbb{M}_{+}\right)} & =\left(\frac{2}{\pi}\right)^{3} \int d^{4} u \overline{G_{1}(u)} G_{2}(u) \\
& =\left(g_{1}, g_{2}\right)_{\mathscr{L}^{2}(\$)} .
\end{aligned}
$$

This leads to a natural isomorphism between the space $\mathscr{L}^{2}(\mathbb{S})$ and the Hilbert space $\mathscr{L}^{2}\left(\mathbb{M}_{4}\right)$. Another choice of the phase in the relation between $G$ and $g$ (2.41) would do the same job. But our choice is most convenient for the purpose of holomorphic extensions.

Finally we are interested in the Szegö kernel for $\mathbb{M}_{4}$. We set

$$
K^{S}\left(w_{1}, w_{2}\right)=K_{w_{1}}^{S}\left(w_{2}\right)=2^{-4}\left[\left(w_{1}-\bar{w}_{2}\right)^{2}\right]^{-2} .
$$


If $g(X) \in \mathscr{L}^{2}(\mathbb{S})$ possesses the holomorphic extension $f_{+}(Z)$ of its positive part $g_{+}(X)(\operatorname{see}(1.86))$

$$
f_{+}(Z)=\left(\overline{K_{Z}^{S}}, g\right)_{\mathscr{L}^{2}(\$)}
$$

then (2.41) and (2.42) imply

$$
\begin{aligned}
f_{+}(Z(w)) & =\left(\frac{2}{\pi}\right)^{3} \int_{\mathbb{M}_{4}} d^{4} u K^{S}(Z(w), X(u)) g(X(u))|\operatorname{det}(E-i U)|^{-4} \\
& =[\operatorname{det}(E-i W)]^{2}\left(\overline{K_{w}^{S}}, G\right)_{\mathscr{L}^{2}\left(\mathbb{M}_{4}\right)}
\end{aligned}
$$

In (2.45) we may replace $G(u)$ by $G_{+}(u)$ that is obtained from (2.41) by inserting the positive part $g_{+}(X)$ of $g(X)$. It follows that

$$
G_{+}(u)=\lim _{v \rightarrow 0 \text { in } \mathrm{L}}\left(\overline{K_{w}^{S}}, G\right)_{\mathscr{L}^{2}\left(\mathbb{M}_{4}\right)}, \quad w=u+i v .
$$

The functions $G_{+}(u)$ form the subspace $\mathscr{L}_{+}^{2}\left(\mathbb{M}_{4}\right)$ of $\mathscr{L}^{2}\left(\mathbb{M}_{4}\right)$.

For any function $G \in \mathscr{L}_{+}^{2}\left(\mathbb{M}_{4}\right)$ the holomorphic extension into $\mathbb{T}$ is obtained by the scalar product with $\overline{K_{w}^{S}},\left(\overline{K_{w}^{S}}, G\right)$. We notice that $K_{w}^{S}$ itself is in $\mathscr{L}_{+}^{2}\left(\mathbb{M}_{4}\right)$ whenever $v=\operatorname{Im} w \neq 0$. In fact, we have

$$
\left(\overline{K_{w_{1}}^{S}}, \overline{K_{w_{2}}^{S}}\right)_{\mathscr{L}^{2}\left(\mathbb{M}_{4}\right)}=\overline{K_{w_{2}}^{S}\left(w_{1}\right)}=K^{S}\left(w_{1}, w_{2}\right)
$$

and therefore

$$
\left\|K_{w}^{S}\right\|_{\mathscr{L}^{2}\left(\mathbb{M}_{4}\right)}=K^{S}(w, w)^{\frac{1}{2}}<\infty
$$

\subsection{Distributions Over Real Minkowski Space}

We define the space of test functions $\mathscr{D}_{\mathscr{L}^{2}}\left(\mathbb{M}_{4}\right)$ [8] to consist of all infinitely differentiable functions $G(u)$ for which the norms

$$
\|G\|_{m, 2}=\left\{\sum_{l \leqq m} \int_{\mathbb{M}_{4}}\left|D_{u}^{l} G(u)\right|^{2} d^{4} u\right\}^{\frac{1}{2}}
$$

are finite for all orders $m$. Here and in the sequel $D_{u}^{l}$ denotes the differential operator

$$
D_{u}^{l}=\prod_{\mu=0}^{3}\left(\frac{\partial}{\partial u_{\mu}}\right)^{l_{\mu}}, \quad|l|=\sum_{\mu} l_{\mu} .
$$

It is easy to see that $K_{w}^{S}$ lies in $\mathscr{D}_{\mathscr{L}^{2}}\left(\mathbb{M}_{4}\right)$ whenever $\operatorname{Im} w \neq 0$. To see this one expresses the differentiation with respect to $u$ by differentiation with respect to $w$ and uses (2.47), (2.48). The linear continuous functionals on $\mathscr{D}_{\mathscr{L}^{2}}\left(\mathbb{M}_{4}\right)$ form the dual space $\mathscr{D}_{\mathscr{L}^{2}}^{\prime}\left(\mathbb{M}_{4}\right)$ which is a subspace of the space $\mathscr{S}^{\prime}\left(\mathbb{M}_{4}\right)$ of tempered distributions over $\mathbb{M}_{4}$. Let $\Phi(u)$ be a distribution of $\mathscr{D}_{\mathscr{L}^{2}}^{\prime}\left(\mathbb{M}_{4}\right)$. Then the extension of the scalar product in $\mathscr{L}^{2}\left(\mathbb{M}_{4}\right)$ to test functions and distributions in the case

$$
F(w)=\left(\overline{K_{w}^{S}}, \Phi\right)
$$


yields a holomorphic function in $\mathbb{T}$. We would like to define a positive part $\Phi_{+}(u)$ of $\Phi(u)$ such that

$$
F(w)=\left(\overline{K_{w}^{S}}, \Phi_{+}\right)
$$

tends to $\Phi_{+}(u)$ in an appropriate topology whenever $\operatorname{Im} w \rightarrow 0$ in $\mathbb{L}$. In the subsequent section we shall then study the holomorphic functions $F(w)$ as elements of $\mathscr{H}_{n, q}(\mathbb{T})$.

First we notice that (2.41) defines a continuous mapping of $\mathscr{E}(\mathbb{S})$ into $\mathscr{D}_{\mathscr{L}^{2}}\left(\mathbb{M}_{4}\right)$. For the proof of this statement we insert (2.41) into (2.49) and use Leibniz's rule. Then we verify first that

$$
[\operatorname{det}(E-i U)]^{2} D_{u}^{m}[\operatorname{det}(E-i U)]^{-2}
$$

is bounded over $\mathbb{M}_{4}$ for each $m$, and second that for the infinitely differentiable map $U \rightarrow X=X(U)$ each derivative is bounded, too. Finally $g(X)$ and its derivatives can be estimated by their supremum. We display the different estimates.

Evaluating the determinant yields

$$
\operatorname{det}(E-i U)=\left(1-i u_{0}\right)^{2}+\sum_{k=1}^{3} u_{k}^{2} .
$$

For fixed $R^{2}=u_{0}^{2}+\Sigma u_{k}^{2}$ the inequalities

$$
1+2 R^{2} \leqq|\operatorname{det}(E-i U)|^{2} \leqq\left(1+R^{2}\right)^{2}
$$

are easily established. Moreover

$$
D_{u}^{m}[\operatorname{det}(E-i U)]^{-2}=P_{|m|}(u)[\operatorname{det}(E-i U)]^{-2-|m|}
$$

where $P_{|m|}(u)$ is a polynomial in the vector components of $u$ of maximal degree $|m|$. Therefore for some constant $C_{m}$

and

$$
\left|P_{|m|}(u)\right| \leqq C_{m}\left(1+2 R^{2}\right)^{\frac{1}{2}|m|}
$$

$$
\left|[\operatorname{det}(E-i U)]^{2} D_{u}^{m}[\operatorname{det}(E-i U)]^{-2}\right| \leqq C_{m} .
$$

The map (2.6) gives

where

$$
\begin{aligned}
X(U) & =(E-i U)^{-1}(E+i U) \\
& =2[\operatorname{det}(E-i U)]^{-1}(E-i \tilde{U})-E
\end{aligned}
$$

Therefore

$$
\tilde{U}=u_{0} E-\sum_{k=1}^{3} u_{k} \sigma_{k}
$$

$$
\begin{aligned}
\left|\frac{\partial^{|m|} x_{i j}}{\prod_{\mu} \partial^{m} u_{\mu}}\right| & =\left|[\operatorname{det}(E-i U)]^{-1-|m|} Q_{|m|+1}(u)\right| \\
& \leqq C_{m}^{\prime}
\end{aligned}
$$

for any matrix element of $X \cdot Q_{|m|+1}(u)$ denotes a polynomial of maximal degree $|m|+1$, and an analogous estimate as in (2.54) is used. 
Finally we have

$$
\begin{aligned}
\int d^{4} u\left|D_{u}^{l} G(u)\right|^{2} & \leqq\left(\frac{\pi}{2}\right)^{3} \sup _{\mathbb{M}_{+}}\left|[\operatorname{det}(E-i U)]^{2} D_{u}^{l} G(u)\right|^{2} \\
& \leqq\left(\sum_{k \leqq l} A_{k} \sup _{\mathrm{S}}\left\{D_{X}^{k} g(X) \mid\right)^{2}\right.
\end{aligned}
$$

with positive constants $A_{k}$ that are independent of $g$. This completes the proof.

Hence $\mathscr{D}_{\mathscr{L}^{2}}^{\prime}\left(\mathbb{M}_{4}\right)$ maps continuously into $\mathscr{E}^{\prime}(\mathbb{S})$. Namely, let $\Phi(u)$ $\mathscr{D}_{\mathscr{L}^{2}}^{\prime}\left(\mathbb{M}_{4}\right), G(u) \in \mathscr{D}_{\mathscr{L}^{2}}\left(\mathbb{M}_{4}\right)$ and $g$ be defined by $(2.41)$. Then

$$
(G, \Phi)_{\mathscr{L}^{2}\left(\mathbb{M}_{4}\right)}=(g, \varphi)_{\mathscr{L}^{2}(\$)}
$$

defines a distribution $\varphi \in \mathscr{E}^{\prime}(\mathbb{S})$. If $g$ is the Szegö kernel $\overline{K_{Z}^{S}}$ then

$$
f(Z)=\left(\overline{K_{Z}^{S}}, \varphi\right)=\left(\overline{K_{Z}^{S}}, \varphi_{+}\right)
$$

tends to $\varphi_{+}(X)$ in the sense of the $\mathscr{E}^{\prime}(\mathbb{S})$ topology when $Z \rightarrow X \in \mathbb{S}$. Explicitly we may write for an arbitrary testfunction $g \in \mathscr{E}(\mathbb{S})$

$$
\left(g, \lim _{Z \rightarrow X}\left(\overline{K_{Z}^{S}}, \varphi\right)\right)=\left(g, \varphi_{+}\right)
$$

and the limit can be realized as the limit of partial sums of the generalized Fourier expansion (1.73).

If we want to carry this limit theorem over to Minkowski space, we must change the space of distributions. It is most convenient to consider tempered distributions. It is obvious that any infinitely differentiable and rapidly decreasing function $G(u)$ on Minkowski space maps onto a function of $\mathscr{E}(\mathbb{S})$ under (2.41). This mapping is moreover continuous from $\mathscr{S}\left(\mathbb{M}_{4}\right)$ into $\mathscr{E}(\mathbb{S})$. For an explicit proof of this statement one makes use of estimates analogous to (2.51) - (2.59). Therefore the space $\mathscr{E}^{\prime}(\mathbb{S})$ maps continuously into the space $\mathscr{S}^{\prime}\left(\mathbb{M}_{4}\right)$ of tempered distributions over Minkowski space. It may happen that different distributions of $\mathscr{E}^{\prime}(\mathbb{S})$ map on the same tempered distribution. An example for this behaviour is the distribution $\delta(-X)$. It is obtained from $\delta(X)$ (Section 1.8) by translation with $-E$ and has support only at $-E$. This distribution maps on the trivial tempered distribution.

Hence the holomorphic extension (2.50)

$$
F(w)=\left(\frac{2}{\pi}\right)^{3} \int K^{S}(w, u) \Phi(u) d^{4} u
$$

with $\Phi \in \mathscr{D}_{\mathscr{L}^{2}}^{\prime}\left(\mathbb{M}_{4}\right)$ has a boundary value $\Phi_{+}(u)$ that is assumed in the sense of the tempered topology. This solves our problem posed at the beginning of this section. However, a deeper insight is gained if we compare our result with an approach based on Fourier and Laplace transformations. As a tempered distribution $\Phi$ can be considered as the 
Fourier transform of another tempered distribution

and similarly

$$
\Phi(u)=\int d^{4} t e^{i t u} \hat{\Phi}(t)
$$

$$
\Phi_{+}(u)=\int d^{4} t e^{i t u} \hat{\Phi}_{+}(t) .
$$

Since $\Phi$ was assumed to be in $\mathscr{D}_{\mathscr{L}^{2}}^{\prime}\left(\mathbb{M}_{4}\right)$, it follows that $\hat{\Phi}(t)$ is realized by a slowly increasing and locally square integrable function. Therefore we can decompose $\hat{\Phi}(t)$ uniquely into the sum of two tempered distributions by

$$
\hat{\Phi}(t)=\theta_{\mathbb{U}}(t) \hat{\Phi}(t)+\left(1-\theta_{\mathbb{L}}(t)\right) \hat{\Phi}(t)
$$

with $\theta_{\mathbb{L}}(t)$ the characteristic function of the forward light cone

$$
\theta_{\mathbb{I}}(t)=\left\{\begin{array}{ll}
1 & t \in \overline{\mathbb{L}} \\
0 & t \notin \overline{\mathbb{L}}
\end{array} .\right.
$$

Due to the theorem quoted in Section 2.1 we have

$$
\hat{\Phi}_{+}(t)=\theta_{\mathbb{L}}(t) \hat{\Phi}(t)
$$

almost everywhere. By the same theorem we have that $F(w)$ is the Laplace transform of $\hat{\Phi}_{+}(t)$ for $\operatorname{Im} w \neq 0$

$$
F(w)=\int d^{4} t e^{i t w} \hat{\Phi}_{+}(t)
$$

as a proper integral. By means of the Laplace transformation of the characteristic function of the forward light cone

$$
\begin{aligned}
\tau(w)= & (2 \pi)^{-4} \int d^{4} t e^{i t w} \theta_{\mathbb{L}}(t) \\
= & \left(\frac{2}{\pi}\right)^{3} K^{S}(w, 0)
\end{aligned}
$$

we can reexpress $F(w)$ as a convolution integral

$$
F(w)=\int d^{4} u \tau(w-u) \Phi(u) .
$$

The two formulae (2.63) and (2.71) are obviously identical.

If $\Phi(u)$ is a general tempered distribution, this decomposition of $\hat{\Phi}(t)$ is no longer unique. In this case we represent $\Phi(u)$ as

$$
\Phi(u)=D_{u}^{m}[\operatorname{det}(E-i U)]^{k} G(u)
$$

where $G(u)$ is square integrable [8]. Then the function

$$
F(w)=D_{w}^{m}[\operatorname{det}(E-i W)]^{k}\left(\overline{K_{w}^{S}}, G\right)_{\mathscr{L}^{2}\left(\mathbb{M}_{4}\right)}
$$

is holomorphic in $\mathbb{T}$ and possesses

$$
D_{u}^{m}[\operatorname{det}(E-i U)]^{k} G_{+}(u)
$$

as distributional boundary value which is assumed in the tempered distribution topology. The non-uniqueness of the decomposition (2.66) 
for tempered distributions $\Phi(u)$ is reflected by the fact that certain polynomials in $u$ can be added to

$$
[\operatorname{det}(E-i U)]^{k} G(u)
$$

without changing $\Phi(u)$ nor the square integrability of $G(u)$.

\subsection{Holomorphic Extensions as Elements of Hilbert Spaces}

The estimate (2.40) and a well-known theorem [10] assure us that the elements $F(w)$ of $\mathscr{H}_{n, q}(\mathbb{T})$ possess tempered distributions $\Phi(u)$ as boundary values on Minkowski space, such that $F$ is the Laplace transform of the inverse Fourier transform $\hat{\Phi}(t)$ of $\Phi(u)$. A characterization of this distributional boundary value as a derivative of a certain order of a square integrable function is easy to obtain from Theorem $\mathrm{A}$ (Section 1.7). We are therefore interested in the opposite problem. Given a tempered distribution $\Phi(u)$ that possesses a holomorphic extension in T, we want to find out for which labels $n$ and $q F(w)$ is an element of $\mathscr{H}_{n, q}(\mathbb{T})$.

First we write $\Phi(u)$ as in (2.72)

$$
\Phi(u)=D_{u}^{m}[\operatorname{det}(E-i U)]^{k} G(u)
$$

where $G$ is square integrable and can moreover be chosen such that its inverse Fourier transform $\hat{G}(t)$ has support in the forward light cone. Then we have as holomorphic extension in $\mathrm{T}$

$$
F(w)=D_{w}^{m}[\operatorname{det}(E-i W)]^{k}\left(\overline{K_{w}^{S}}, G\right)_{\mathscr{L}^{2}\left(\mathbb{M}_{4}\right)}
$$

Proceding now as in the alternative form of Theorem B in Section 1.7, we apply Schwarz's inequality

$$
|F(w)|^{2} \leqq\|G\|_{\mathscr{L}^{2}\left(\mathbb{M}_{4}\right)}^{2} D_{w}^{m} D_{\bar{w}}^{m}|\operatorname{det}(E-i W)|^{2 k} K^{S}(w, w)
$$

Inserted into (2.33) this yields

$$
\begin{aligned}
\|F\|_{n, q}^{2} \leqq c\|G\|_{\mathscr{L}^{2}\left(\mathbb{M}_{4}\right)}^{2} \int|d W|[\operatorname{det}(\operatorname{Im} W)]^{n-4} & \\
\cdot & |\operatorname{det}(E-i W)|^{-2 q} D_{w}^{m} D_{\bar{w}}^{m}|\operatorname{det}(E-i W)|^{2 k} K^{S}(w, w)
\end{aligned}
$$

Our task consists in estimating this integral.

We have

$$
\begin{aligned}
D_{w}^{m} D_{\bar{w}}^{m}|\operatorname{det}(E-i W)|^{2 k} & K^{S}(w, w) \\
& =R_{2|m|+4 k}(w, \bar{w})\left[(\operatorname{Im} w)^{2}\right]^{-2-2|m|}
\end{aligned}
$$

with a polynomial $R_{2|m|+4 k}(w, \bar{w})$ of maximal degree $2|m|+4 k$. We put

$$
R^{2}=u_{0}^{2}+v_{0}^{2}+\sum_{k=1}^{3}\left(u_{k}^{2}+v_{k}^{2}\right)
$$


and obtain for large $R$

$$
1+2 R^{2} \leqq|\operatorname{det}(E-i W)|^{2} .
$$

Hence we have for all $w$ with a certain constant $C_{m, k}$

$$
R_{2|m|+4 k}(w, \bar{w}) \leqq C_{m, k}|\operatorname{det}(E-i W)|^{2|m|+4 k} .
$$

The right hand side of (2.77) is majorized this way by

$c C_{m, k}\|G\|_{\mathscr{L}^{2}\left(\mathbb{M}_{4}\right)}^{2} \int|d W|[\operatorname{det}(\operatorname{Im} W)]^{n^{\prime}-4}|\operatorname{det}(E-i W)|^{-2 q+2|m|+4 k}$

with

$$
n^{\prime}=n-2-2|m| \text {. }
$$

Therefore the integral (2.77) converges whenever

$$
\begin{aligned}
n^{\prime} & \geqq 4 \\
2 n^{\prime}-2 q+2|m|+4 k & \leqq 0 .
\end{aligned}
$$

Rewriting the conditions (2.84) in terms of $n$ we obtain

$$
\begin{aligned}
& n \geqq 2|m|+6 \\
& q \geqq n-|m|-2+2 k
\end{aligned}
$$

as sufficient conditions for the finiteness of $\|F\|_{n, q}$. Of course these conditions need not be necessary. The first condition involves $n$ only, that means: $n$ is determined primarily by the local singularities of $\Phi(u)$.

\section{Applications}

\subsection{Conformally Covariant Fields}

The connection between classical field theory and the representation theory of the Poincaré group is well known. In a similar spirit we can consider the distributional boundary values $\Phi(u)$ of a holomorphic function $F(w)$ as parts of a classical conformally covariant field [11]. Classical fields are vector valued functions over Minkowski space with values in a space that carries a representation of the little group for the origin in Minkowski coordinate space. In our case the little group is that subgroup of $S U(2,2)$ that maps $w=0$ into $w^{\prime}=0$ under $(2.15)$. It consists of dilations, the group $\operatorname{SL}(2, C)$, special conformal transformations, the central elements, and the products of all these. In this section it is most convenient to set $q=0$ and to consider only the spaces $\mathscr{H}_{n, 0}(\mathbb{T})$.

First we put

$$
R=\lambda^{-\frac{1}{2}} E, \quad \lambda \text { real }
$$

in (2.20) and obtain the dilation operators

$$
T_{\lambda} \Phi(u)=\lambda^{-n} \Phi\left(\lambda^{-1} u\right)
$$


with the generator

$$
-\left.i \frac{d T_{\lambda}}{d \lambda}\right|_{\lambda=1} \Phi(u)=i\left(n+u_{\mu} \frac{\partial}{\partial u_{\mu}}\right) \Phi(u) .
$$

Following a customary use we call $n$ the "scale dimension" of the field $\Phi(u)$. The fact that $n$ is limited to $n \geqq 2$, whereas the "canonical scale dimension" of a scalar field is one, could cause us to try an "analytic continuation" of our approach in $n$ to values smaller than two. Comparison with the analogous problem in the case of the group $S U(1,1)$ shows that this attempt might force us to change the metric and switch to another series of representations.

The special conformal transformations are obtained from (2.35)

$$
T_{t} \Phi(u)=\left(1+2 t u+t^{2} u^{2}\right)^{-n} \Phi\left(u^{\prime}\right)
$$

where $u^{\prime}$ is defined in (2.30). In infinitesimal form we get

$$
\begin{aligned}
K_{\mu} \Phi(u) & =-\left.i \frac{\partial}{\partial t^{\mu}} T_{t}\right|_{t=0} \Phi(u) \\
& =i\left[2 n u_{\mu}-\left(u^{2} g_{\mu v}-2 u_{\mu} u_{v}\right) \frac{\partial}{\partial u_{v}}\right] \Phi(u) .
\end{aligned}
$$

The representation of the little group of $u=0$ is trivial for the special conformal group

$$
K_{\mu} \Phi(0)=0 .
$$

Infinitesimal elements of $S L(2, C)$ yield

$$
M_{\mu v} \Phi(u)=i\left[u_{\mu} \frac{\partial}{\partial u^{v}}-u_{v} \frac{\partial}{\partial u^{\mu}}\right] \Phi(u) .
$$

The corresponding representation of the little group for $u=0$ is again trivial, our fields are scalar (spin zero). Since scalar fields are too narrow a class for reasonable applications we are forced to generalize our concepts.

Classical fields with non-zero spin are in fact obtained from the other members of the series $d_{0}$ [1]. These spaces consist of functions $f(X, Y, Z)$ which depend on complex matrices $X, Y, Z$. $f$ is assumed to be holomorphic (respectively antiholomorphic) for fixed $X$ and $Y$ and for $Z$ in $\mathbb{D}$. It is moreover a homogeneous polynomial of degree $2 j_{1}\left(2 j_{2}\right)$ in the elements of the first row of the $2 \times 2$ matrix $X(Y)$. Therefore we have in particular (see (1.44))

$$
f(X, Y, Z)=\sum_{q_{1}=-j_{1}}^{+j_{1}} \sum_{q_{2}=-j_{2}}^{+j_{2}} D_{j_{1} q_{1}}^{j_{1}}(X) D_{j_{2} q_{2}}^{j_{2}}(Y) f_{q_{1} q_{2}}(Z)
$$


Let $K_{1,2}$ be matrices and $d \mu(K)$ the normalized Haar measure of $S U(2)$. $N_{1,2}$ are the matrix functions (1.14) of $Z$. A sesquilinear form for these functions can then be introduced by

$$
\begin{gathered}
(f, g)=c^{\prime} \int \overline{f\left(\overline{N_{1} K_{1}}, N_{2} K_{2}, Z\right)} g\left(\overline{N_{1} K_{1}}, N_{2} K_{2}, Z\right) \\
\quad \cdot\left[\operatorname{det}\left(E-Z^{+} Z\right)\right]^{n-4} d \mu\left(K_{1}\right) d \mu\left(K_{2}\right)|d Z| \\
=\frac{c^{\prime}}{\left(2 j_{1}+1\right)\left(2 j_{2}+1\right)} \int\left\{\sum_{q_{1} q_{2}} \overline{f_{q_{1} q_{2}}(Z)} g_{q_{1} q_{2}}(Z)\right\} \\
\cdot D_{j_{1} j_{1}}^{j_{1}}\left(N_{1}^{2}\right) D_{j_{2} j_{2}}^{j_{2}}\left(N_{2}^{2}\right)\left[\operatorname{det}\left(E-Z^{+} Z\right)\right]^{n-4}|d Z| .
\end{gathered}
$$

With the same notations as in (1.21)-(1.23) we define

$$
T_{M} f(X, Y, Z)=[\operatorname{det}(C Z+D)]^{-n} f\left(\overline{\left(A+B Z^{+}\right)} X,(C Z+D) Y, Z^{\prime}\right) \text {. }
$$

For $n \geqq 4+2 j_{1}+2 j_{2}$ integral, the sesquilinear form (3.9) obviously converges and defines a scalar product that is invariant. Correspondingly it determines a Hilbert space of functions $f(X, Y, Z)$ that carries a unitary irreducible representation of $S U(2,2)$. By similar arguments as used for the case $j_{1}=j_{2}=0$ in Section 1.4 we may extend the validity of the scalar product till $n=3+2 j_{1}+2 j_{2}$. But an attempt to go down further fails for the same reasons as in the case $j_{1}=j_{2}=0$.

The theory of these representations and their distributional boundary values can be carried through similarly as in the case $j_{1}=j_{2}=0$. The scale dimension of the boundary limit $\Phi_{q_{1} q_{2}}(u)$ is $n-j_{1}-j_{2}$. The representation of the little group for $u=0$ contains the finite dimensional representation $\left(j_{1}, j_{2}\right)$ of $S L(2, C)$. If we compare these results with classical fields [11] we should have

$$
n=1+2 j_{1}+2 j_{2}
$$

for canonical free fields. For vector currents $\left(j_{1}=j_{2}=\frac{1}{2}\right)$ and for the energy-momentum tensor $\left(j_{1}=j_{2}=1\right)$ we find instead

$$
n=2+2 j_{1}+2 j_{2} \text {. }
$$

We can therefore apply our formalism not to these basic objects but rather to products of fields and currents.

\subsection{Operator Products and Inclusive Reactions}

One calls inclusive reactions those processes where two elementary particles hit and thereby produce a bulk of new particles, a fraction of which is only observed. Examples are

or

$$
\text { proton }+ \text { proton } \rightarrow \text { pion }+ \text { anything }
$$

$$
\text { photon }+ \text { proton } \rightarrow \text { anything . }
$$


The cross section for these processes depends on the total four momentum $P$ of the unobserved objects. This momentum lies in the forward light cone. By means of a Fourier transformation, after splitting off an appropriate kinematical factor, these cross sections can be related with the diagonal matrix elements of products of local operators. These matrix elements are assumed to be tempered distributions. Therefore they possess a holomorphic extension into the tube domain.

As an example we study the first process quoted above. In the centreof-momentum system of the ingoing protons the cross section is [12]

$$
\frac{d \sigma}{d^{3} q}=\frac{(2 \pi)^{3} M^{2}}{4 E p q^{0}} \int d^{4} u e^{i q u} \frac{1}{4} \sum_{\text {Spins }} \text { in }\left\langle p_{1}, p_{2}\left|j_{\pi}(0) j_{\pi}(u)\right| p_{1}, p_{2}\right\rangle_{\text {in }}
$$

with the proton momenta

$$
p_{1}=(E, 0,0, p), \quad p_{2}=(E, 0,0,-p), \quad p_{1}^{2}=p_{2}^{2}=M^{2}
$$

and the pion momentum

$$
q=p_{1}+p_{2}-P .
$$

Using the completeness of physical states and the spectrum condition we get

$$
e^{i\left(p_{1}+p_{2}\right) u}{ }_{\text {in }}\left\langle p_{1}, p_{2}\left|j_{\pi}(0) j_{\pi}(u)\right| p_{1}, p_{2}\right\rangle_{\text {in }}=\int d^{4} P e^{i P u} A\left(p_{1}, p_{2}, P\right)
$$

where the support of $A$ in the variable $P$ lies in the forward light cone. Therefore the function $F(w)$ defined as the Laplace transform

is holomorphic in $\mathbb{T}$.

$$
F(w)=\int d^{4} P e^{i P w} A\left(p_{1}, p_{2}, P\right)
$$

A formula for the norm $\|F\|_{n, q}$ can only be implicitly given for general index $q$ (see (2.33))

$$
\|F\|_{n, q}^{2}=\int d^{4} P \int d^{4} P^{\prime} M_{n, q}\left(P, P^{\prime}\right) A\left(p_{1}, p_{2}, P\right) A\left(p_{1}, p_{2}, P^{\prime}\right) .
$$

Note that $A$ is a real function. $M_{n, q}$ is then defined by the integral

$$
M_{n, q}\left(P, P^{\prime}\right)=c \int_{\mathbb{M}_{4}} d^{4} u \int_{\mathbb{L}} d^{4} v e^{i P(u+i v)-i P^{\prime}(u-i v)}\left(v^{2}\right)^{n-4}|\operatorname{det}(E-i W)|^{-2 q}
$$

For $q=0$ a simple expression results (for $q<0 M_{n, q}$ is obtained from this expression by derivation)

$$
M_{n, 0}=8 \pi(n-1) !(n-2) ! \delta\left(P-P^{\prime}\right)\left(P^{2}\right)^{-n+2} .
$$

Hence the norm of $F$ is

$$
\|F\|_{n, 0}^{2}=8 \pi(n-1) !(n-2) ! \int d^{4} P\left(P^{2}\right)^{-n+2}\left(A\left(p_{1}, p_{2}, P\right)\right)^{2} .
$$

Whenever the unobserved set of particles may consist of a single (stable) particle this norm does obviously not exist since $A$ contains a term 
$\delta\left(P^{2}-m^{2}\right)$. Apart from the deuteron which we want to neglect, a stable particle with baryon number two is known not to exist. However, in the photon induced process quoted at the beginning the proton itself is such a particle. In these cases we must try $q>0$. Evaluation of the integral (3.17) is not easy for these $q$.

In the customary treatment of an inclusive process like inelastic electron-proton scattering one performs an asymptotic expansion of the operator product at the light cone [13]

$$
j(x) j(0) \underset{x^{2} \rightarrow 0}{\cong} \sum_{\alpha} c_{\alpha}(x) F_{\alpha}(0)
$$

where $F_{\alpha}(0)$ is a local operator and $c_{\alpha}(x)$ a homogeneous or associate homogeneous distribution whose degree is determined by dimensional arguments. The distribution $c_{\alpha}(x)$ of maximal singularity dominates the function $A$ in the "scaling limit" $P^{2} \rightarrow \infty$. This light cone singularity is quite unlikely to dominate the on-shell processes like the one-pion inclusive process discussed here [14]. Instead we obtain an integrability condition (3.19) for $A$ which accounts for the high- $P$ behaviour in a more implicit way but on the other hand reflects the influence of all types of singularities.

\section{References}

1. Graev, M.L.: Dokl. Akad. Nauk SSSR 98, 517 (1954).

2. Esteve, A., Sona, P. G.: Nuovo Cimento 32, 473 (1964).

3. Rühl, W.: The Lorentz Group and Harmonic Analysis, eq. (2-29) New York: W.A. Benjamin, Inc. 1970.

4. Meschkowski,H.: Hilbertsche Räume mit Kernfunktion. Grundl. Math. Wiss. Bd. 113, Berlin-Göttingen-Heidelberg: Springer 1962.

5. Köthe, G.: Math. Z. 57, 13 (1952), J. r. angew. Math. 191, 30 (1953).

6. Tillmann, H. G.: Math. Z. 59, 61 (1953), 76, 5 (1961), 77, 125 (1961).

7. Neumark, M.A.: Normierte Algebren, p. 224, Berlin: VEB Deutscher Vlg. der Wissenschaften 1959.

8. Schwartz, L.: Théorie des Distributions, Paris: Hermann 1966.

9. Streater, R. F., Wightman, A. S.: PCT, Spin and Statistics, and All That, Theorem 2-9, New York: W. A. Benjamin, Inc. 1964.

10. Ref. 9, Theorem 2-10.

11. Mack, G., Salam, A.: Ann. Phys. 53, 174 (1969).

12. Mueller, A. H.: Phys. Rev. D 2, 2963 (1971).

13. Frishman, Y.: Ann. Phys. 66, 373 (1971) and further literature quoted there.

14. Müller,V.F., Rühl,W.: University of Trier-Kaiserslautern preprint, November 1971.

W. Rühl

Universität Trier-Kaiserslautern

Fachbereich Physık

D-6750 Kaiserslautern

Pfaffenbergstraße 95

Germany 